\title{
Lagrangian Mechanics of Fractional Order, Hamilton-Jacobi Fractional PDE and Taylor's Series of Nondifferentiable Functions
}

\author{
A short version of this paper is (will be) published in the journal \\ Chaos, Solitons and Fractals
}

\author{
Guy JUMARIE \\ Department of Mathematics \\ University of Québec at Montréal \\ P.O. Box 8888, Downtown Station \\ Montréal, Qc \\ H3C $3 P 8$ \\ Canada \\ jumarie.guy@uqam.ca
}

The paper proposes an extension of the Lagrange analytical mechanics to deal with dynamics of fractal nature. Rirst of all, by using fractional difference, one introduces a slight modification of the Riemann-Liouville derivative definition, which is more consistent with self-similarity by removing the effect of the initial value, and then for the convenience of the reader, one gives a brief background on the Taylor's series of fractional order $f(x+h)=E_{\alpha}\left(h^{\alpha} D_{x}{ }^{\alpha}\right) f(x)$ of nondifferentiable function, where $E_{\alpha}$ is the MittagLeffler function. The Lagrange characteristics method is extended for solving a class of nonlinear fractional partial differential equations. All this material is necessary to solve the problem of fractional optimal control and mainly to find the characteristics of its fractional Hamilton-Jacobi equation, therefore the canonical equations of optimality. Then fractional Lagrangian mechanics is considered as an application of fractional optimal control. Proposals for a differential geometry of fractional order are outlined. In this framework, the use of complex-valued variables, as Nottale did it, appears as a direct consequence of the irreversibility of time, and it is shown that there is consistency with the Lorentz transformation .

Keywords: Fractional PDE, Lagrange characteristics, fractional Taylor series, fractal mechanics, fractal spacetime, fractional Hamilton-Jacobi equation, fractional differential geometry. 


\section{Introduction}

A problem which has attracted the attention of many scientists, is to prove the Schrödinger equation from a set of consistent axioms instead of taking it itself as an axiom [4]. One can partition papers on this topics in two main parts: those which deal with the problem in the classical probabilistic framework, and those which directly refer to the fractal nature of quantum space-time, or again, the zooming feature of quantum space-time. For instance one can find an approach via rotating vector [12], an approach via Fisher information [11] and an approach via the central limit theorem [38]. The Three (Kings) Magi who recognized that the key to clarify the mysteries of quantum mechanics is to take for granted its fractal nature are Nottale [35-37], Ord [39] and Nelson [34] who probably provided some suggestions to El Nashie to build up his E infinite theory [9].

The main idea is that the basic equations of physics, Schrödinger, Klein-Gordon and Dirac equations, would be geodesic equations in a fractal space-time framework. The quantum feature would be a direct consequence of the fractal geometry of spacetime.

But as pointed out by Nottale, a function which is continuous everywhere but nowhere differentiable is not, cannot be replicated; in other words, and it is our conclusion, it would necessarily exhibits random or random-like features. As a result, the literature on fractal on the one hand and on fractional stochastic processes on the other hand, are more or less related, and to some extend, one could claim that one of the the genuine reasons for expanding fractional calculus is its use in the analysis of dynamical systems subject to fractional Brownian motion $[5,8,14,17]$.

This could perhaps explain why the first derivation (proof) of the Shrödinger equation has been obtained in setting the problem in a stochastic framework involving random motion. In this way of thought, one could ask whether the Shrödinger equation is not merely a direct consequence of the Itö's lemma? Some concerns which might be of some interests to be investigated are the followings.

Modeling of nondifferentiability. It may be of interest to get more insight in the modeling of nondifferentiability by means of Brownian motion [15,43], and this would help us to generalize the formalism by means of Brownian motion of order $n$. The motivation for such a generalization is that fractional Brownian motion of order $n$ takes account of collisions between $n$ particles. In quantum mechanics, we assume that only collisions between two particles are relevant, but if we use the formalism of quantum mechanics to deal with other topics like mathematical finance [14,21], for instance, it is likely that other values $n>2$ may be quite meaningful.

Complex variables in physics. Another topic of reflection is the use of complex variables in quantum mechanics. For some authors, it is merely a practical way to deal simultaneously with two variables, and nothing more. For others on the contrary, it is compulsory and one cannot expand quantum mechanics without it.

With these objectives in mind, in quite a natural way, we are led to try to construct a Lagrangian mechanics of fractional order and our purpose in the following is to display the results which we have so obtained.

Loosely speaking, the paper comprises two main parts: the first one which provides new results on fractional calculus and their application to fractional partial differential equations, and the second one which deals with optimal control and mechanics. It is organized as follows.

(i) First of all, since we have in mind some more or less implicite reference to Nottale's fractal spacetime theory, we shall give a brief background on the latter (section 2), and by this way the reader will be in a better position to compare with our approach. 
(ii) To some extent, there is some inconsistency between the Riemann-Liouville definition of fractional derivative and self-similarity, which are caused by the nonzero initial conditions of the considered function. In order to circumvent this pitfall, many authors use the so-called Caputo fractional derivative, which at the extreme says that if you want to have the first derivative of a function, you must before have at hand its second derivative! In order to cope with this difficulty, we propose here a slight modification of the Riemann-Liouville definition, which yields zero as the fractional derivative of a constant (section 3)

(iii) This new fractional derivative definition will allow us to provide a sound basis for the fractional Taylor's series which we have proposed recently, and at the same time, we take the opportunity to contribute some new results about (section 4), and more especially we shall display some fractional derivative chain rules.

(iv) We shall then have at hand all the material which is necessary to generalize the Lagrange's method of characteristics for solving some linear fractional partial differential equations (section 5) and the approach will be extended to nonlinear fractional partial differential equations (section 6). We shall so have at hand a method for solving FPDE, which is much more general than those we have at hand presently and which apply to some special cases only $[1,2,10,16,42,44]$

(v) This prerequisite is necessary to deal with fractional optimal control and two different models are considered depending upon the differentiability of the functions so involved. The fractional canonical equations of optimality are obtained via fractional variational calculus, and their relation with the fractional Hamilton-Jacobi equation is clarified (sections 7 and 8).

(vi) Applying these results above, we shall consider the dynamics of a particle defined by a fractional velocity and an action function expressed as an integral with respect to $(d t)^{\alpha}$.

(vii) Then we shall examine in which way we could build up a differential geometry of fractional order, and how the results of the paper are related to the Lorentz equations. Lastly, the conclusion will suggest some open problems for future research. notations

In the following we shall use at will and for convenience, depending upon the case, the

and

$$
D^{\alpha} f(x)=f^{(\alpha)}(x)=\frac{d^{\alpha} f(x)}{d x^{\alpha}}
$$

$$
D_{x}^{\alpha} f(x, y)=f_{x}^{(\alpha)}(x, y)=\frac{\partial^{\alpha} f(x, y)}{\partial x^{\alpha}}=\partial_{x}^{\alpha} f(x, y)
$$

for the fractional derivative and the fractional partial derivative respectively. Also, we shall write

$$
\Gamma(1+\alpha)=: \quad \alpha !, \quad \alpha \geq 0
$$

where $\Gamma(x)$ is the Eulerian gamma function, even when $\alpha$ is not an integer.

To come into contact with the core of the paper, the hasty reader may skip the sections 5 and 6 which explain how one can obtain the solution of the Hamilton-Jacobi equation.

\section{Short Background on Quantum Fractal Space-Time}

\subsection{Summary of the key ideas}

Loosely speaking, Nottale [35-37] started from the following ideas to expand his theory.

(i) Firstly, he characterizes a real-valued non-differential function by a complex-valued velocity $v_{c}$ (different from the usual $v$ ) which is a complex combination of its backward derivative (derivative on the left) with its forward derivative (derivative on the right).

(ii) And secondly, he assumes that a mechanical system is defined by a Lagrangian $L\left(x, v_{c}, t\right)$ which explictly involves this complex-valued velocity.

Loosely speaking, all the axioms of classical quantum mechanics, including Schrödinger equation are replaced by the above two ones, and it is not a second ranking success! 
For the sake of simplicity and to shorten the present paper, we shall once more consider one-dimensional systems only, but the reader will generalize easily to three co-ordinates.

(i) Basically, a co-ordinate $x(t)$ is of fractal nature, and can be split in the form

$$
x(t)=\bar{x}(t)+\xi(t),
$$

where $\bar{x}(t)$ is the mean value (ensemble average) of $x(t),\langle x(t)\rangle=\bar{x}(t)$, and $\xi(t)$ is a term which takes account of the fluctuation around $\bar{x}(t)$. A small increment $d x(t)$ of $x(t)$ is

$$
d x(t)=d \bar{x}(t)+d \xi(t),
$$

where $d \bar{x}(t)$ is the differential of $\bar{x}(t)$, and $d \xi(t)$ is a non-deterministic term such that

$$
\begin{gathered}
\langle d \xi(t)\rangle=0, \\
\left\langle(d \xi(t))^{2}\right\rangle=\lambda d t, \quad \lambda>0 .
\end{gathered}
$$

(ii) $x(t)$ is not differentiable and thus has two derivatives: a derivative on the left $\dot{x}_{-}$, referred to as the backward derivative, and a derivative on the right $\dot{x}_{+}$, the forward derivative. Nottale combines them to define the complex velocity

$$
\widetilde{v}:=(1 / 2)\left(\dot{x}_{+}+\dot{x}_{-}\right)-i(1 / 2)\left(\dot{x}_{+}-\dot{x}_{-}\right) \text {. }
$$

(iii) This being so, given a function $f(x, t)$, its differential is defined by the expression

$$
d f=\partial_{t} f d t+\partial_{x} f d x+(1 / 2) \partial_{x x} f(d x)^{2}
$$

of which the mean value is (according to (2.2) and (2.4))

$$
\langle d f\rangle=\partial_{t} f d t+\partial_{x} f d \bar{x}+(1 / 2) \lambda \partial_{x x} f d t
$$

and as a result, one is led to define the total derivative of $f(x, t)$ by the expression

$$
d f / d t=\left(\partial_{t}+v \partial_{x}+(1 / 2) \lambda \partial_{x x}\right) f \text {. }
$$

with $v:=d \bar{x} / d t$.

This expression holds on the right $(+)$ and on the left (-), and Nottale combines them to introduce the complex scale-covariant derivative.

$$
d_{s c} / d t:=\left(\partial_{t}+\widetilde{v} \partial_{x}-i(1 / 2) \lambda \partial_{x x}\right) .
$$

where $V$ is defined by (3.5).

\subsection{Derivation (Proof) of Schrödinger equation}

Approach via complex Lagrangian

(i) One assumes that a (one-dimensional) mechanical system is defined by the Lagrangian $L(x, \tilde{v}, t)$. One can then refer to its action $S$

$$
S:=\int_{t_{1}}^{t_{2}} L(x, \widetilde{v}, t) d t,
$$

the optimization of which will provide its dynamical trajectory.

In the special case when $L=(1 / 2) m \widetilde{v}^{2}-v V(x)$, where $V(x)$ is the potential function in the usual sense of this term, the dynamical equation reads

$$
m\left(d_{s c} \tilde{v} / d t\right)=-\partial_{x} V .
$$

(ii) According to well known result of optimization theory (this is the understanding of the author!), one has the equality

$$
\tilde{v}=(1 / m) \partial_{x} S .
$$

(iii) Next, Nottale defines the function $\psi$ by the expression

therefore, by virtue of (2.11),

$$
\psi:=\exp \{i S / m \lambda\}
$$

$$
\tilde{v}=-i \lambda \partial_{x}(\ln \psi) .
$$

Substituting this result into (2.11) one obtains the equality 
which reduces to

$$
\partial_{x} V=\operatorname{Im} d_{s c}\left(\partial_{x x} \ln \psi\right) / d t
$$

$$
\begin{aligned}
\frac{d_{s c}}{d t} \tilde{v}= & -\lambda \frac{\partial}{\partial x}\left(i \frac{\partial}{\partial t} \ln \psi+\frac{\lambda}{2} \frac{\partial_{x} \psi}{\psi}\right) \\
= & (-1 / m) \partial_{x} V .
\end{aligned}
$$

Integrating with respect to time yields the equation

$$
(1 / 2) \lambda^{2} \partial_{x x} \psi+i \lambda \partial_{t} \psi-(V / m) \psi=0,
$$

which is exactly the Schrödinger equation.

\section{Further remarks and comments}

Relation with Nelson modeling. As a matter of fact, the complex derivative (2.5) has been suggested to Nottale by Nelson [34] who characterized a position vector $x(t)$ by two stochastic differential equations depending upon whether $d t>0$ or $d t<0$, namely

$$
d x(t)=f_{+}(x(t)) d t+d \xi_{+}(t), \quad d t>0,
$$

and

$$
d x(t)=f_{-}(x(t)) d t+d \xi_{-}(t), \quad d t<0,
$$

where $\xi(t)$ is a Brownian motion which satisfies the averaging condition

$$
\begin{aligned}
\left\langle d \xi_{+, i}(t) d \xi_{+, j}(t)\right\rangle & =2 Q \delta_{i j} d t, \\
\left\langle d \xi_{-, i}(t) d \xi_{i, j}(t)\right\rangle & =-2 Q \delta_{i j} d t .
\end{aligned}
$$

with $Q$ denoting a positive diffusion coefficient.

Let $p(x, t)$ denote the probability density of $x(t)$. Then acccording to (2.15) and (2.16) it satisfies the two diffusion equations

$$
\partial p / \partial t+\operatorname{div}\left(p f_{+}\right)=Q \Delta p
$$

and

$$
\partial p / \partial t+\operatorname{div}\left(p f_{-}\right)=-Q \Delta p .
$$

These equations suggest to introduce the new velocity vector

$$
U_{x}:=\left(f_{+}+f_{-}\right) / 2, U_{y}:=\left(f_{+}-f_{-}\right) / 2
$$

which provides the continuity equation

$$
\partial p / \partial t+\operatorname{div}\left(p U_{x}\right)=0
$$

in addition with

$$
\operatorname{div}\left(p U_{y}\right)-Q \Delta p=0 .
$$

Here is the divergence between Nelson's and Nottale's approaches. Nelson goes farther by using a mixture of equations (2.22) and (2.23) with Newton equation, whilst Nottale introduces the complex velocity vector (2.5) associated with (2.21).

On the significance of the complex velocity. According to Nottale, the assumption that the system is driven by a Lagrangian $L(x, \widetilde{v}, t)$, which depends upon the complex velocity $\widetilde{v}$, can be supported as follows.

Usually, the Lagrangian is a function of the variable $x$ and of its derivative $\dot{x}$. When $x$ is not differentiable, then in quite a natural way, one is led to assume that the Lagrangian $L\left(x, \dot{x}_{+}, \dot{x}_{-}, t\right)$ is a function of both $\dot{x}_{+}$and $\dot{x}_{-}$. This being the case, one can prove that, if one combines $\dot{x}_{+}$and $\dot{x}_{-}$in the form of the complex velocity $V$, then the Euler-Lagrange equations derived from this Lagrangian has exactly the form of the classical one. In other words, the use of $L(x, V, t)$ is fully supported by the requirement of covariance or similarly, of invariance of the equations.

In the following, we shall examine in which way this formalism is or can be related to our fractional calculus, and we shall show that, to some extent, the use of complex variables appears 
as a direct result of irreversibility of time.. To this end, first of all, we shall introduce a new modified Riemann-Liouville definition of fractional derivative to cope with some problems which arise with nonzero initial conditions.

\section{Fractional Derivative Revisited}

\subsection{Fractional derivative via fractional difference}

Definition 3.1 Let $f: \mathfrak{R} \rightarrow \mathfrak{R}, x \rightarrow f(x)$, denote a continuous (but not necessarily differentiable) function, and let $h>0$ denote a constant discretization span. Define the forward operator $F W(h)$ (the symbol:= means that the left side by the right one)

$$
F W(h) . f(x):=f(x+h) ;
$$

then the fractional difference of order $\alpha, \alpha \in \mathfrak{R}, 0<\alpha \leq 1$, of $f(x)$ is defined by the expression

$$
\begin{aligned}
\Delta^{\alpha} \cdot f(x) & :=(F W-1)^{\alpha} \cdot f(x) \\
& =\sum_{k=0}^{\infty}(-1)^{k}\left(\begin{array}{l}
\alpha \\
k
\end{array}\right) f[x+(\alpha-k) h],
\end{aligned}
$$

and its fractional derivative of order $\alpha$ (F-derivative in the following) is

$$
f^{(\alpha)}(x)=\lim _{h \downarrow 0} \frac{\Delta^{\alpha} f(x)}{h^{\alpha}} .
$$

This definition is close to the standard definition of derivative (calculus for beginners), and as a direct result, the $\alpha$-th derivative of a constant is zero.

Proposition 3.1 Assume that the function $f(x)$ in the Definition 3.1 is not a constant, then its fractional derivative of order $\alpha$ is defined by the following expression [26,29,30,31],

$$
f^{(\alpha)}(x):=\frac{1}{\Gamma(-\alpha)} \int_{0}^{x}(x-\xi)^{-\alpha-1} f(\xi) d \xi, \quad \alpha<0 .
$$

For positive $\alpha$, one will set

$$
\begin{gathered}
f^{(\alpha)}(x)=\left(f^{(\alpha-1)}(x)\right)^{\prime}, \quad 0<\alpha<1, \\
f^{(\alpha)}(x):=\left(f^{(\alpha-n)}(x)\right)^{(n)}, \quad n \leq \alpha<n+1, \quad n \geq 2 .
\end{gathered}
$$

When $f(x)$ is a constant function, the expression (3.4) does not apply in the sense that it is not consistent with (3.3), and in order to be consistent with (3.3), we shall define its F-derivative as being zero.

Proof. The proof can be obtained by using Laplace transform and $Z$-transform and then making $h$ tends to zero. By this way, one can show that (3.3) and (3.8) have the same Laplace transform. See for instance [17].

\subsection{Modified Riemann-Liouville derivative}

The first definition of F-derivative which has been proposed in the literature is the so-called Riemann-Liouville definition which reads as follows

Definition 3.1 (Riemann-Liouville) Let $f: \mathfrak{R} \rightarrow \mathfrak{R}, x \rightarrow f(x)$, denote a continuous function. Its fractional derivative of order $\alpha$ is defined by the expressions (3.4), (3.5) and (3.6) for every $f($.

But this definition gives rise to the following problem. If one makes $f(x)$ constant in (3.4) one finds that the $\alpha$-th derivative of a constant is $x^{-\alpha} / \Gamma(1-\alpha)$ that is to say is different from zero. To circumvent this failure, some authors $[3,6]$ proposed to use rather the definition

$$
f^{(\alpha)}(x):=\frac{1}{\Gamma(1-\alpha)} \int_{0}^{x}(x-\xi)^{-\alpha} f^{\prime}(\xi) d \xi, \quad 0<\alpha<1,
$$




$$
f^{(\alpha)}(x):=\frac{1}{\Gamma(n+1-\alpha)} \int_{0}^{x}(x-\xi)^{n-\alpha} f^{(n+1)}(\xi) d \xi, \quad n<\alpha<n+1,
$$

but we are reluctant to do so, for two reasons. First, according to this definition, the $\alpha$-th derivative, $0<\alpha<1$, would be defined for differentiable functions only, whilst on the contrary, the very reason of using fractional derivative is exactly to deal with nondifferentiable functions! And second, at the extreme, this expression says that if we want to get the first derivative of a function, we must before to have its second derivative. We believe that a definition via finite difference can be of help to solve this pitfall.

An alternative to the Riemann-Liouville definition of fractional derivative

In order to circumvent this drawback, we propose the following alternative to the RiemannLiouville definition of F-derivative

Definition 3.2 (Riemann-Liouville definition revisited). Refer to the function of Proposition 3.1. Then its fractional derivative of order $\alpha$ is defined by the expression

$$
f^{(\alpha)}(x):=\frac{1}{\Gamma(-\alpha)} \int_{0}^{x}(x-\xi)^{-\alpha-1}(f(\xi)-f(0)) d \xi, \quad \alpha<0 .
$$

For positive $\alpha$, one will set

$$
\begin{aligned}
f^{(\alpha)}(x) & =\left(f^{(\alpha-1)}(x)\right)^{\prime}, \quad 0<\alpha<1, \\
& =\frac{1}{\Gamma(1-\alpha)} \frac{d}{d x} \int_{0}^{x}(x-\xi)^{-\alpha}(f(\xi)-f(0)) d \xi,
\end{aligned}
$$

and

$$
f^{(\alpha)}(x):=\left(f^{(n)}(x)\right)^{(\alpha-n)}, \quad n \leq \alpha<n+1, \quad n \geq 1 .
$$

We shall refer to this fractional derivative as to the modified Riemann Liouville derivative.

First of all, it is of order to point out that the definitions 3.1 and 3.2 are quite equivalent. Moreover our definition and the so-called Caputo's definition yield the same result, but here we deal with the $\alpha$-th derivative only, $0<\alpha<1$, without referring to the derivative itself!. Once again, the Caputo definition exhibits the embarrassing feature that the first derivative is defined by means of the second one.

With this definition, the Laplace transform $L\{\}$ of the F-derivative is

$$
L\left\{f^{(\alpha)}(x)\right\}=s^{\alpha} L\{f(x)\}-s^{\alpha-1} f(0), \quad 0<\alpha<1 .
$$

Let us point out that Mandelbrot had already recognized the troublesome effects of the initial conditions in the Riemann-Liouville definition of derivative, and to rectify this defect, he proposed to use the definition [31] see also [32,33]

$$
f^{(\alpha)}(x):=\frac{1}{\Gamma(-\alpha)} \int_{-\infty}^{x}(x-\xi)^{-\alpha-1} f(\xi) d \xi, \quad \alpha<0 .
$$

\subsection{Self-similarity, fractal, fractional derivative and initial value}

We believe that this problem which so occurs with the customary Riemann-Liouville definitive is not too much surprising, and that on the contrary, it is quite right so. Indeed the basic motivation of fractional derivative is to deal with fractional function which themselves are selfsimilar. But a self-similar function $x(t)$ is necessary such that $x(t)=0$. In other words, to avoid any inconsistency between the Riemann-Liouville definition on the one hand, and the property of self-similar functions on the other hand we have to remove the constant term. We can get some more insight in this feature as follows.

In the stochastic framework, a self-similar process $x(t)$ of index $\alpha$ is a process which satisfies the condition

$$
x(\rho t) \stackrel{\text { law }}{=} \rho^{\alpha} x(t), \quad \rho>0,
$$


that is to say $x(\rho t)$ and $\rho^{\alpha} x(t)$ have the same probability density function. As a result, one has the equality

$$
x(t) \stackrel{\text { law }}{=} t^{\alpha} x(1), \quad t>0
$$

therefore

$$
d x \stackrel{\text { law }}{=}(d t)^{\alpha} x(1), \quad t=0,
$$

and we then arrive at fractional derivative in quite a natural way. Shortly, self-similar processes are fractional and they are analyzed by means of fractional calculus. But according to the equation (3.13), one should have $x(0)=0$ with the probability one.

In other words, the functions which we are considering when we work with fractional derivative, are those which satisfy the condition $x(0)=0$, and as a result we should remove any nonzero initial condition in the Riemann-Liouville definition.

\section{Background on Taylor's Series of Fractional Order}

\subsection{Main definition}

A generalized Taylor expansion of fractional order (F-Taylor series in the following) reads as follows

Proposition 4.1 Assume that the continuous function $f: \Re \rightarrow \Re, x \rightarrow f(x)$ has fractional derivative of order $k \alpha$, for any positive integer $k$ and any $\alpha, 0<\alpha<1$, then the following equality holds, which is

$$
f(x+h)=\sum_{k=0}^{\infty} \frac{h^{\alpha k}}{\Gamma(1+\alpha k)} f^{(\alpha k)}(x), \quad 0<\alpha \leq 1 .
$$

where $f^{(\alpha k)}$ is the derivative of order $\alpha k$ of $f(x)$.

With the notation

$$
\Gamma(1+\alpha k)=: \quad(\alpha k) !,
$$

one has the formula

$$
f(x+h)=\sum_{k=0}^{\infty} \frac{h^{\alpha k}}{(\alpha k) !} f^{(\alpha k)}(x), \quad 0<\alpha \leq 1
$$

which looks like the classical one.

Indication on the proof. Refer to the forward shift operator $F W(h)$ defined by the relation (3.1), then it is easy to verify that it satisfies the equality

$$
D_{h}^{\alpha} F W(h) f(x)=F W(h) D_{x}^{\alpha} f(x) .
$$

In other words, formally, $F W(h)$ is defined by the fractional differential equation

$$
D_{h}^{\alpha} F W(h)=F W(h) D_{x}^{\alpha},
$$

and according to the definitions 3.1 and 3.2, its solution is

$$
F W(h)=E_{\alpha}\left(h^{\alpha} D_{x}^{\alpha}\right),
$$

where $E_{\alpha}(x)$ denotes the Mittag-Leffler function defined by the expression

$$
E_{\alpha}(x):=\sum_{k=0}^{\infty} \frac{x^{k}}{\Gamma(1+\alpha k)} .
$$

We then have the equality

$$
f(x+h)=E_{\alpha}\left(h^{\alpha} D_{x}^{\alpha}\right) f(x) .
$$

therefore (4.1) and (4.2). For further details see [23,24]

Corollary 4.1 Assume that $m<\alpha \leq m+1, m \in N-\{0\}$ and that $f(x)$ has derivatives of order $k$ (integer), $1 \leq k \leq m$, then, integrating the serial expansion 


$$
f^{(m)}(x+h)=\sum_{k=0}^{\infty} \frac{h^{k(\alpha-m)}}{\Gamma[1+k(\alpha-m)]} D^{k(\alpha-m)} f^{(m)}(x), \quad m<\alpha \leq m+1,
$$

with respect to $h$ yields

$$
f(x+h)=\sum_{k=o}^{m} \frac{h^{k}}{k !} f^{(k)}(x)+\sum_{k=1}^{\infty} \frac{h^{(k \beta+m)}}{\Gamma(k \beta+m+1)} f^{(k \beta+m)}(x), \quad \beta:=\alpha-m .
$$

In the special case when $m=1$, one has

$$
f(x+h)=f(x)+h f^{\prime}(x)+\sum_{k=1}^{\infty} \frac{h^{k \beta+1}}{\Gamma(k \beta+2)} f^{(k \beta+1)}(x), \quad \beta:=\alpha-1 .
$$

Mc-Laurin series of fractional order

Let us make the substitution $h \leftarrow x$ and $x \leftarrow 0$ into (4.1), we so yobtain the fractional McLaurin series

$$
f(x)=\sum_{k=0}^{\infty} \frac{x^{\alpha k}}{\Gamma(1+\alpha k)} f^{(\alpha k)}(0), \quad 0<\alpha \leq 1 .
$$

As a direct application of the fractional Taylor's series, one has the following

Corollary 4.2 Assume that $f(x)$, in Proposition 4.1, is $\alpha$-th differentiable, then the following equalities hold, which are

$$
f^{(\alpha)}(x)=\lim _{h \downarrow 0} \frac{\Delta^{\alpha} f(x)}{h^{\alpha}}=\Gamma(1+\alpha) \lim _{h \downarrow 0} \frac{\Delta f(x)}{h^{\alpha}}, \quad 0<\alpha<1 .
$$

and

$$
f^{(\alpha)}(x)=\Gamma[1+(\alpha-m)] \lim _{h \downarrow 0} \frac{\Delta f^{(m)}(x)}{h^{\alpha-m}}, \quad m<\alpha<m+1 .
$$

\section{Useful relations}

The equation (4.1) provides the useful relation

$$
\Delta^{\alpha} f \cong \Gamma(1+\alpha) \Delta f, \quad 0<\alpha<1,
$$

or in a differential form $d^{\alpha} f \cong \Gamma(1+\alpha) d f$, between fractional difference and finite difference.

Corollary 4.3 The following equalities hold, which are

$$
\begin{gathered}
D^{\alpha} x^{\gamma}=\Gamma(\gamma+1) \Gamma^{-1}(\gamma+1-\alpha) x^{\gamma-\alpha}, \quad \gamma>0, \\
(u(x) v(x))^{(\alpha)}=u^{(\alpha)}(x) v(x)+u(x) v^{(\alpha)}(x), \\
(f[u(x)])^{(\alpha)}=f_{u}^{\prime}(u) u^{(\alpha)}(x), \\
=f_{u}^{(\alpha)}(u)\left(u_{x}^{\prime}\right)^{\alpha} .
\end{gathered}
$$

Proof. These derivatives are straightforward consequences of the equality

$$
d^{\alpha} x(t)=\Gamma(1+\alpha) d x(t) .
$$

For instance one has $d(u v)=v d u+u d v$, and multiplying both sides by $\alpha ! /(d t)^{\alpha}$ direct yields (4.12). A similar rationale is used to obtain the other formulas. In (4.13) $f(u)$ is assumed to be differentiable, whilst in (4.14) it is $\alpha$-th differentiable.

Corollary 4.4 Assume that $f(x)$ and $x(t)$ are two $\mathfrak{R} \rightarrow \mathfrak{R}$ functions which both have derivatives of order $\alpha, 0<\alpha<1$, then one has the chain rule

$$
f_{t}^{(\alpha)}(x(t))=\Gamma(2-\alpha) x^{\alpha-1} f_{x}^{(\alpha)}(x) x^{(\alpha)}(t) \quad .
$$

Proof. The $\alpha$-th derivative of $x$ provides the equality

$$
d^{\alpha} x=\frac{1}{(1-\alpha) !} x^{1-\alpha}(d x)^{\alpha} .
$$

which allows us to write 
whereby the result.

$$
\begin{aligned}
d^{\alpha} f & =f_{x}^{(\alpha)}(d x)^{\alpha} \\
& =f_{x}^{(\alpha)}(x)(1-\alpha) ! x^{\alpha-1} d^{\alpha} x
\end{aligned}
$$

\subsection{Further results and remarks}

On the suitable fractional derivative definition to be selected

(i) With the definition 2.2, the solution of the equation (4.3) is exactly the Mittag-Leffler function. Indeed, let us consider the fractional differential equation

$$
D_{t}^{\alpha} x(t)=-\lambda x(t), x(0)=x_{0} .
$$

Its solution can be obtained as follows. Using the definition of fractional derivative, equation (3.10), the equation (4.17)) can be explicitly re-written in the form

$$
\frac{1}{\Gamma(1-\alpha)} \frac{d}{d t}\left(\int_{0}^{t} \frac{x(\tau)-x(0)}{(t-\tau)^{\alpha}} d \tau\right)=-\lambda x(t),
$$

or

$$
\int_{0}^{t}(t-\tau)^{-\alpha}(x(\tau)-x(0)) d \tau=-\lambda \Gamma(1-\alpha) \int_{0}^{t} x(\tau) d \tau
$$

or (by an obvious change of variable)

$$
t^{1-\alpha} \int_{0}^{1}(1-u)^{-\alpha}(x(t u)-x(0)) d u=-\lambda \Gamma(1-\alpha) t \int_{0}^{1} x(t u) d u .
$$

The presence of $t^{\alpha}$ in this equation on the one hand, and the solution $\exp \{-\lambda t\}$ of the equation $d p / d t=-\lambda p$ on the other hand, suggest to look for a solution in the form

$$
x(t)=\sum_{k=0}^{\infty} x_{k}\left(t^{\alpha}\right)^{k} .
$$

Substituting (4.19) into (4.18), one obtains the equation

$$
x_{k+1}=-\lambda \frac{\Gamma(\alpha k+1)}{\Gamma(\alpha k+\alpha+1)} x_{k}, \quad k \geq 0 .
$$

which yields

$$
x_{k}=\frac{(-\lambda)^{k}}{\Gamma(\alpha k+1)} x_{0}
$$

therefore

$$
\begin{aligned}
x(t) & =x_{0} \sum_{k=0}^{\infty} \frac{\left(-\lambda t^{\alpha}\right)^{k}}{\Gamma(k \alpha+1)}, \\
& =x_{0} E_{\alpha}\left(-\lambda t^{\alpha}\right),
\end{aligned}
$$

An alternative is to take the Laplace transform of the equation (4.17) to obtain, with our modified Riemann-Liouville derivative

$$
s^{\alpha} X(s)-s^{\alpha-1} x(0)=-\lambda X(s)
$$

where $X(s)$, which is the Laplace transform of $x(t)$, yields the Mittag-Leffler function.

On the differentiability of $f(x)$

(ii) As it is obvious, the series (4.1) applies to nondifferentiable functions, whilst (4.9) refers to differentiable functions.

(iii) Assume that $\alpha=1 / N, N$ integer, in the F-Taylor series (4.1); then when $k=N$ we come across the first derivative. Nevertheless this does not mean that there is some inconsistency somewhere, but rather it is the meaning of these equations which must be clarified. Indeed, because of the presence of $h^{\alpha}, h$ is restricted to be positive, $h>0$; and as a result, all the 
derivatives involved in the F-Taylor series (4.1), either they are fractional or not, are derivatives on the right.

In other words, at first glance, in the Nottale's formulation, the velocity of the particle should be defined by the right derivative $\dot{x}_{+}$only.

Modeling irreversibility of time

Assume that $f($.$) is a function f(t)$ of time; then according to the above comments, the FTaylor series of $f(t+\Delta t)$ holds for positive $\Delta t$ only. This property can be thought of as a practical describing of the irreversibility of time.

Self-similarity and fractional Mc-Laurin series

Assume that $x(t)$ is a self-similar function with the similarity index $H$, clearly

$$
x(a t)=a^{H} x(t), \quad a>0, \quad 0<H<1 .
$$

then, one can check easily that the fractional Mc-Laurin series of order $\alpha, 0<\alpha<1$, of both sides are the same.

Indeed the equality (4.24) provides easily

$$
x^{(k \alpha)}(u)=a^{H-k \alpha} x^{(k \alpha)}(t), \quad u:=a t,
$$

therefore

$$
\begin{aligned}
x(u) & =\sum_{k=0}^{\infty} \frac{u^{k \alpha}}{(k \alpha) !}\left[x^{(k \alpha)}(u)\right]_{u=0} \\
& =\sum_{k=0}^{\infty} \frac{(a t)}{(k \alpha) !} a^{H-k \alpha}\left[x^{(k \alpha)}(t)\right]_{t=0} \\
& =a^{H} x(t) .
\end{aligned}
$$

Relation with previous results in the literature

(iv) Osler [40] has previously proposed a generalization of Taylor's series in the complex plane, in the form

$$
f(z)=\alpha \sum_{k=-\infty}^{k=+\infty} \frac{f^{(\alpha k)}\left(z_{0}\right)}{\Gamma(1+\alpha k)}\left(z-z_{0}\right)^{\alpha k},
$$

which, in the useful case when $f(z)$ is continuous at $z_{0}$, reduces to

$$
f(z)=\alpha \sum_{k=0}^{k=+\infty} \frac{f^{(\alpha k)}\left(z_{0}\right)}{\Gamma(1+\alpha k)}\left(z-z_{0}\right)^{\alpha k} .
$$

So, in the special case when $z=z_{0}$, this equality (4.27) would provide

$$
f\left(z_{0}\right)=\alpha f\left(z_{0}\right) \text { ? }
$$

(vi) More recently Kolwankar and Gangal [27,28] proved the so-called "local fractional Taylor expansion"

$$
f(x+h)=\sum_{k=0}^{m} \frac{h^{k}}{k !} f^{(k)}(x)+\frac{f^{(\alpha)}(x)}{\Gamma(1+\alpha)} h^{\alpha}+R_{\alpha}(h), \quad m<\alpha<m+1 .
$$

where $R_{\alpha}(h)$ is a remainder, which is negligible when compared with the other terms. This is exactly our series (4.8), but here we give an explicit expression for $R_{\alpha}(h)$, namely

$$
R_{\alpha}(h)=\sum_{k=2}^{\infty} \frac{h^{(k \beta+m)}}{\Gamma(k \beta+m+1)} f^{(k \beta+m)}(x), \quad \beta:=\alpha-m .
$$

Nevertheless, it is relevant to point out that these authors do not use the Riemann-Liouville expression of derivative as we did it, but rather define the later as the limit of a quotient involving the increment of the function on the one hand, and a so-called coarse grained mass or $\alpha$-mass of 
a subset which is generally fractal. Loosely speaking the function is fractal because it is defined on a set which itself is fractal.

\subsection{Fractional derivative of the Dirac delta function}

The solution of the equation

$$
d x=f(t)(d t)^{\alpha}, \quad t \geq 0, \quad x(0)=x_{0}, \quad 0<\alpha \leq 1,
$$

is defined by the following result

Lemma 4.1 Let $f(t)$ denote a continuous function, then the solution of the equation (4.30) is defined by the equality (see for instance [22])

$$
\int_{0}^{t} f(\tau)(d \tau)^{\alpha}=\alpha \int_{0}^{t}(t-\tau)^{\alpha-1} f(\tau) d \tau \quad, \quad 0<\alpha \leq 1 .
$$

Application to the fractional derivative of the Dirac delta function

On using the equation (4.11) on the one hand, and extending well known definition on the other hand, we shall define the fractional derivative of the Dirac delta function by the equality

$$
\int \delta^{(\alpha)}(\tau) f(\tau)(d \tau)^{\alpha}=-\int \delta(\tau) f^{(\alpha)}(\tau)(d \tau)^{\alpha}, \quad 0<\alpha \leq 1
$$

and the equation (4.31), direct will yields

$$
\int \delta^{(\alpha)}(\tau) f(\tau)(d \tau)^{\alpha}=-\alpha t^{\alpha-1} f^{(\alpha)}(0), \quad 0<\alpha \leq 1
$$

\subsection{Integration with respect to $(d t)^{\alpha}$}

The solution of the equation

$$
d x=f(t)(d t)^{\alpha}, \quad t \geq 0, \quad x(0)=x_{0},
$$

is defined by the following result:

Lemma 4.2 Let $f(t)$ denote a continuous function, then the solution of the equation (4.32) is defined by the equality

$$
\int_{0}^{t} f(\tau)(d \tau)^{\alpha}=\alpha \int_{0}^{t}(t-\tau)^{\alpha-1} f(\tau) d \tau \quad, \quad 0<\alpha \leq 1 .
$$

Some examples

(i) On making $f(\tau)=1$ in (4.35) one obtains

$$
\int_{0}^{t} \tau^{\gamma}(d \tau)^{\alpha}=\frac{\Gamma(\alpha+1) \Gamma(\gamma+1)}{\Gamma(\alpha+\gamma+1)} t^{\alpha+\gamma}, \quad 0<\alpha \leq 1
$$

(ii) Assume now that $f(t)$ is the Dirac delta generalized function $\delta(t)$, then one has

$$
\int_{0}^{t} \delta(\tau)(d \tau)^{\alpha}=\alpha t^{\alpha-1}, \quad 0<\alpha \leq 1 .
$$

With this prerequisite, we can now address the problem of solving some fractional partial differential equations.

\section{Lagrange Method for Solving Fractional Linear PDE}

\subsection{Statement of the problem}

Our purpose in the following is to determine the solution of the FPDE

$$
f(x, y, u) \frac{\partial^{\alpha} u}{\partial x^{\alpha}}+g(x, y, u) \frac{\partial u}{\partial y}=h(x, y, u), \quad 0<\alpha<1,
$$

where $u: \mathfrak{R}^{2} \rightarrow \mathfrak{R},(x, y) \rightarrow u(x, y)$, is subject to the initial condition

$$
u(x, o)=u_{0}(x) .
$$

In the special case when $\alpha=1$, the most useful approach to solve for $u(x, y)$ is the Lagrange technique which introduces the auxiliary system

$$
\frac{d x}{f}=\frac{d y}{g}=\frac{d u}{h}
$$


and to look for two integral functions $\Phi_{1}(x, y, u)=$ const and $\Phi_{2}(x, y, u)=$ const which provide the general solution in the form $F\left(\Phi_{1}, \Phi_{2}\right)$. In the following, we shall show how this method can be modified to solve the equation (5.1). To this end, we need a preliminary result on systems of fractional (ordinary) differential equations.

\subsection{On Systems of fractional differential equations}

Let us consider the system

$$
\begin{gathered}
\dot{x}(t)=f_{1}(x, y, t), \quad x(0)=x_{0}, \\
y^{(\alpha)}(t)=f_{2}(x, y, t), \quad y(0)=y_{0}, \quad 0<\alpha<1,
\end{gathered}
$$

where $t$ denotes time, to fix the thought. We have the following result:

Lemma 5.1. Assume that $\Psi(x, y, t)=$ const is a first integral for the system $(5.4,5.5)$; then it is solution of the following FPDE

$$
f_{1}^{\alpha} \frac{\partial^{\alpha} \Psi}{\partial x^{\alpha}}+f_{2} \frac{\partial \Psi}{\partial y}+\frac{1}{\Gamma(2-\alpha)} t^{1-\alpha} \frac{\partial \Psi}{\partial t}=0
$$

Proof. Applying the operator $E_{\alpha}\left(d x^{\alpha} D_{x}{ }^{\alpha}\right) \exp \left(d y D_{y}\right) \exp \left(d t D_{t}\right)$ to the function $\Psi$, we get the increment

$$
\begin{gathered}
d \Psi=\frac{1}{\alpha !} \frac{\partial^{\alpha} \Psi}{\partial x^{\alpha}}(d x)^{\alpha}+\frac{\partial \Psi}{\partial y} d y+\frac{\partial \Psi}{\partial t} d t \\
d \Psi=\frac{1}{\alpha !} \frac{\partial^{\alpha} \Psi}{\partial x^{\alpha}}(d x)^{\alpha}+\frac{1}{\alpha !} \frac{\partial \Psi}{\partial y} d^{\alpha} y+\frac{1}{\alpha !} \frac{\partial \Psi}{\partial t} d^{\alpha} t \\
d \Psi=\frac{1}{\alpha !}\left(\frac{\partial^{\alpha} \Psi}{\partial x^{\alpha}}\left(\frac{d x}{d t}\right)^{\alpha}+\frac{\partial \Psi}{\partial y} \frac{d^{\alpha} y}{d t^{\alpha}}+\frac{\partial \Psi}{\partial t} \frac{d^{\alpha} t}{d t^{\alpha}}\right)(d t)^{\alpha}
\end{gathered}
$$

and we remark that

$$
\frac{d^{\alpha} t}{d t^{\alpha}}=\frac{1}{\Gamma(2-\alpha)} t^{1-\alpha}
$$

to obtain the equation (5.6).

\subsection{Auxiliary system associated with FPDE}

We now come back to the fractional PDE (5.1), and we denote by $\Phi(x, y, u)$ first integral (function), namely $\Phi(x, y, u)=$ constant. We have the following result:

Lemma 5.2. Let $\Phi(x, y, u)=K$ denote a first integral function for the FPDE (5.1); then it satisfies the FPDE

$$
f \frac{\partial^{\alpha} \Phi}{\partial x^{\alpha}}+g \frac{\partial \Phi}{\partial y}+h \frac{\partial \Phi}{\partial u}=0 .
$$

Proof. (i) Let $d_{x} \Phi$ denote the increment of $\Phi$ along $x$ only. We then have

$$
\begin{aligned}
d_{x} \Phi & =\frac{\partial \Phi}{\partial u} d_{x} u+\frac{1}{\alpha !} \frac{\partial^{\alpha} \Phi}{\partial x^{\alpha}}(d x)^{\alpha} \\
& =\frac{1}{\alpha !} \frac{\partial \Phi}{\partial u} d_{x}^{\alpha} u+\frac{1}{\alpha !} \frac{\partial^{\alpha} \Phi}{\partial x^{\alpha}}(d x)^{\alpha} \\
& =\frac{1}{\alpha !}\left(\frac{\partial \Phi}{\partial u} \frac{\partial^{\alpha} u}{\partial x^{\alpha}}+\frac{\partial^{\alpha} \Phi}{\partial x^{\alpha}}\right),
\end{aligned}
$$

and on expliciting the condition $d_{x} \Phi=0$, we obtain 


$$
\frac{\partial^{\alpha} u}{\partial x^{\alpha}}=-\frac{\Phi_{x}^{(\alpha)}}{\Phi_{u}^{\prime}}
$$

(ii) The same (standard) calculation w.r.t. $y$ yields

therefore, on equating to zero,

$$
d_{y} \Phi=\left(\frac{\partial \Phi}{\partial u} \frac{\partial u}{\partial y}+\frac{\partial \Phi}{\partial y}\right) d y
$$

$$
\frac{\partial u}{\partial y}=-\frac{\Phi_{y}^{\prime}}{\Phi_{u}^{\prime}}
$$

(iii) Substituting (5.10) and (5.11) into (5.1) yields the result (5.9)

Auxiliary system associated with the FPDE

Lemma 5.3. The auxiliary system of partial differential equations associated with the FPDE (5.1) is

$$
\frac{(d x)^{\alpha}}{f}=\frac{d^{\alpha} y}{g}=\frac{(d u)^{\alpha}}{h} \text { - }
$$

Derivation of these equations. The key idea is provided by the similarity between the FPDE (5.6) and (5.9). More explicitly, we re-write (5.9) in the form

$$
\frac{1}{\Gamma(2-\alpha)} t^{1-\alpha}\left(\frac{f^{\alpha}}{h} \frac{\partial^{\alpha} \Phi}{\partial x^{\alpha}}+\frac{g}{h} \frac{\partial \Phi}{\partial y}+\frac{\partial \Phi}{\partial u}\right)=0 .
$$

On comparing (5.13) with (5.6), we are led to make the substitution $t \leftarrow u$ and to set

$$
\begin{aligned}
f_{1}^{\alpha}(x, y, u) & \equiv \frac{u^{1-\alpha} f(x, y, u)}{(1-\alpha) ! h(x, y, u)}, \\
f_{2}(x, y, u) & \equiv \frac{u^{1-\alpha} g(x, y, u)}{(1-\alpha) ! h(x, y, u)} .
\end{aligned}
$$

We then have the associated differential equations (the parallels of (5.4) and (5.5))

$$
\begin{aligned}
\left(\frac{d x}{d u}\right)^{\alpha} & =\frac{u^{1-\alpha} f(x, y, u)}{(1-\alpha) ! h(x, y, u)}, \\
\frac{d^{\alpha} y}{d u^{\alpha}} & =\frac{u^{1-\alpha} g(x, y, u)}{(1-\alpha) ! h(x, y, u)}
\end{aligned}
$$

therefore the associated system (5.12)

\subsection{Some illustrative examples}

Example 5.1 Let us consider the FPDE

$$
u_{t}^{(\alpha)}(x, t)+c u_{x}^{\prime}(x, t)=0, \quad 0<\alpha<1
$$

where $c$ denotes a constant. The associated system is

$$
\frac{(d t)^{\alpha}}{1}=\frac{d^{\alpha} x}{c}=\frac{(d u)^{\alpha}}{0}
$$

therefore the auxiliary equations

$$
\begin{gathered}
d^{\alpha} x=c(d t)^{\alpha}, \\
u_{t}^{\prime}=0,
\end{gathered}
$$

which provide the first integrals

$$
\alpha ! x+c t^{\alpha}=\text { const }
$$

and

$$
u=\text { const }
$$


We then obtain the general solution

$$
u(x, t)=F\left(\Gamma(1+\alpha) x+c t^{\alpha}\right) .
$$

Example 5.2. Let us consider now the FPDE

$$
\left(D_{t}^{2 \alpha}-c^{2} D_{x}^{2}\right) u(x, t)=0, \quad 0<\alpha<1 .
$$

Where $c$ denotes a constant. On re-writing it in the form

we are led to solve the equations

$$
\left(D_{t}^{\alpha}-c D_{x}\right)\left(D_{t}^{\alpha}+c D_{x}\right) u(x, t)=0,
$$

and

$$
u_{t}^{(\alpha)}-c u_{x}^{\prime}=0
$$

$$
u_{t}^{(\alpha)}+c u_{x}^{\prime}=0
$$

and, using the result related to the example (5.1) above direct yield

$$
u(x, t)=F\left(\Gamma(1+\alpha) x+c t^{\alpha}\right)+G\left(\Gamma(1+\alpha) x-c t^{\alpha}\right) .
$$

Example 5.3. We once more consider the FPDE

$$
u_{t}^{(\alpha)}(x, t)=\lambda(x-1) u_{x}^{\prime}(x, t),
$$

with the initial condition $u(x, 0)=x$. The associated auxiliary system is

$$
\frac{(d t)^{\alpha}}{1}=-\frac{d^{\alpha} x}{\lambda(x-1)}=\frac{(d u)^{\alpha}}{0}
$$

therefore the equations

$$
\begin{aligned}
u & =\text { const }, \\
x^{(\alpha)}(t) & =-\lambda(x-1) .
\end{aligned}
$$

We shall solve this equation by using the variation of constant as follows.

(i) Firstly we consider the homogeneous equation

$$
x^{(\alpha)}(t)=-\lambda x(t) .
$$

of which the solution is

$$
x(t)=C E_{\alpha}\left(-\lambda t^{\alpha}\right)
$$

where $C$ denotes a constant.

(ii) A special solution of (5.26) is obtained by assuming that $C$ turns to be a function $C(t)$. We then have

$$
\begin{aligned}
x^{(\alpha)}(t) & =C^{(\alpha)}(t) E_{\alpha}\left(-\lambda t^{\alpha}\right)+C(t) E_{\alpha}^{(\alpha)}\left(-\lambda t^{\alpha}\right) \\
& =C^{(\alpha)}(t) E_{\alpha}\left(-\lambda t^{\alpha}\right)-\lambda C(t) E_{\alpha}\left(-\lambda t^{\alpha}\right)
\end{aligned}
$$

and on substituting into (5.26) we obtain the $\mathrm{C}$-equation

$$
C^{(\alpha)}(t)=\lambda E_{\alpha}^{-1}\left(-\lambda t^{\alpha}\right)
$$

therefore

$$
C(t)=E_{\alpha}^{-1}\left(-\lambda t^{\alpha}\right) .
$$

Substituting $C(t)$ into (5.27), we find that the special sought solution is the unity, in such a manner that the general solution of (5.26) which reads

$$
x=C E_{\alpha}\left(-\lambda t^{\alpha}\right)+1,
$$

provides the first integral

$$
(x-1) E_{\alpha}^{-1}\left(-\lambda t^{\alpha}\right)=\text { const } .
$$

We now come back to the solution of the equation (5.23) which, by using the above results, can be written in the form

$$
u(x, t)=F\left((x-1) E_{\alpha}^{-1}\left(-\lambda t^{\alpha}\right)\right) .
$$


On taking account of the initial condition $u(x, 0)=x$, we find that

$$
F(\xi)=\xi+1
$$

therefore the sought solution

$$
u(x, t)=(x-1) E_{\alpha}^{-1}\left(-\lambda t^{\alpha}\right)+1 .
$$

Example 5.4 The following FPDE

$$
\frac{\partial^{\alpha} u}{\partial t^{\alpha}}-a x \frac{\partial u}{\partial x}+\frac{\lambda x^{2}}{\beta^{2}+x^{2}}=0
$$

with the boundary conditions

$$
u(0, t)=u(x, 0)=1 .
$$

occurs in the study of oscillators subjected to fractional Poissonian noises. In fact, in this probabilistic framework, if one wants to use the customary related notations, then $u(x, t)$ should be rather read $\Phi(u, t), u(x, t) \leftarrow \Phi(u, t)$, which is the characteristic function of a random variable.

The auxiliary system associated with (5.28) is

$$
\frac{(d t)^{\alpha}}{1}=-\frac{d^{\alpha} x}{a x}=-\frac{\beta^{2}+x^{2}}{\lambda x^{2}} \frac{(d u)^{\alpha}}{u}
$$

therefore the two equations

$$
\begin{aligned}
x^{(\alpha)}(t) & =-a x(t), \\
\frac{(d u)^{\alpha}}{u} & =\frac{\lambda}{a} \frac{x d^{\alpha} x}{\beta^{2}+x^{2}} .
\end{aligned}
$$

(i) As usual now, the solution of (5.31) is $x=x_{0} E_{\alpha}\left(-a t^{\alpha}\right)$ and provides the first integral

$$
x E_{\alpha}^{-1}\left(-a t^{\alpha}\right)=\text { const }
$$

(ii) The solution of the equation (5.32) is based on the following two results

First: one has the equality (see the equation (4.12))

$$
(f[g(x)])^{(\alpha)}=f_{g}^{\prime}(g) g^{(\alpha)}(x) .
$$

Second: one has the inference (see the appendix)

$$
\text { If } g^{(\alpha)}(u)=\frac{1}{u} \quad \text { then } \quad g(u)=\frac{u^{\alpha-1}}{(\alpha-1) \Gamma(2-\alpha)},
$$

or again

$$
\int \frac{(d u)^{\alpha}}{u}=\frac{u^{\alpha-1}}{(\alpha-1) \Gamma(2-\alpha)} .
$$

By using these results, we integrate both sides of (5.32) to obtain ( $K$ denotes a constant)

$$
\ln \frac{\left(\beta^{2}+x^{2}\right)^{\lambda / 2 a}}{K}=\frac{u^{\alpha-1}}{(\alpha-1) \Gamma(2-\alpha)},
$$

therefore the first integral (function)

$$
\left(\beta^{2}+x^{2}\right)^{\lambda / 2 a} \exp \left(-\frac{u^{\alpha-1}}{(\alpha-1) \Gamma(2-\alpha}\right)=\text { const } .
$$

(iii) On combining (5.33) and (5.38), the general solution of the equation (5.28) can be written in the form

$$
\left(\beta^{2}+x^{2}\right)^{\lambda / 2 a} \exp \left(-\frac{u^{\alpha-1}}{(\alpha-1) \Gamma(2-\alpha}\right)=F\left(x E_{\alpha}^{-1}\left(a t^{\alpha}\right)\right) .
$$


The explicit expression of $F(x)$ is given by the boundary condition (5.29) and we have eventually

$$
\frac{u^{\alpha-1}-1}{(\alpha-1) \Gamma(2-\alpha)}=\ln \left(\frac{\beta^{2}+x^{2}}{\beta^{2}+x^{2} E_{\alpha}^{-2}\left(a t^{\alpha}\right)}\right)^{\lambda / 2 a} .
$$

Remark that when $\alpha=1$, then $u^{\alpha-1} \cong 1+(\alpha-1) \ln u$, and one has

$$
u(x, t)=\left(\frac{\beta^{2}+x^{2}}{\beta^{2}+x^{2} e^{2 a t}}\right)^{\lambda / 2 a},
$$

which is exactly the solution which we would obtain directly by using the auxiliary system

\section{Lagrange Method for Solving Fractional Nonlinear FPDE}

\section{Statement of the problem}

Our purpose is to obtain the solution of the fractional partial differential equation

$$
F(x, y, u, p, q)=0,
$$

where $F$ is a nonlinear real-valued function, $x \in \mathfrak{R}$ and $y \in \mathfrak{R}$ are two independent variables, $u(x, t)$ is the unknown function to be determined, $p$ is the fractional partial derivative of $u(x, y)$ w.r.t. $x$,

$$
p=\frac{\partial^{\alpha} u}{\partial x^{\alpha}} \equiv \partial_{x}^{\alpha} u \equiv u_{x}^{(\alpha)}, \quad 0<\alpha \leq 1
$$

and $q$ is the partial derivative of $u$ w.r.t. $y ; q=\partial_{y} u=u_{y}$.

Lemma 6.1. Main result: auxiliary system for solution

Solving (6.1) amounts to solve the following system of equations

$$
\frac{(d x)^{\alpha}}{F_{p}}=\frac{d^{\alpha} y}{F_{q}}=\frac{d^{\alpha} u}{p F_{p}+q F_{q}}=-\frac{d^{\alpha} p}{F_{x}^{(\alpha)}+p F_{u}}=-\frac{d^{\alpha} q}{F_{y}+q F_{u}} .
$$

Derivation of the equation (6.2)

(i) The idea is to look for another function $G$ such that

$$
G(x, y, u, p, q)=C,
$$

where $C$ denotes a constant. Strictly speaking, $F$ and $G$ defines $p=\varphi(x, y, u)$ and $q=\psi(x, y, u)$ as functions of $x, y$ and $u$, and then their integration will provide $u$. In order to determine $\mathrm{G}$ we shall write that the following consistency condition is satisfied,

$$
\partial_{y}\left(\partial_{x}^{\alpha} u\right)=p_{y}+p_{u} q=\partial_{x}^{\alpha}\left(\partial_{y} u\right)=q_{x}^{(\alpha)}+q_{u} p
$$

and to this end we need the explicit expressions of the partial derivatives $p$ and $q$, which will be obtained by equating to zero the partial differential $d_{x} F, d_{y} F, d_{u} F$ and similarly for $G$.

(ii) $d_{y} F$ and $d_{u} F$ are obtained by the usual chain rule, for instance

$$
d_{y} F=\left(F_{y}+F_{p} p_{y}+F_{q} q_{y}\right) d y .
$$

Regarding $d_{x} F$, one has (on combining fractional and nonfractional Taylor series)

$$
\begin{aligned}
d_{x} F & =\Gamma^{-1}(1+\alpha) F_{x}^{(\alpha)}(d x)^{\alpha}+F_{p} d_{x} p+F_{q} d_{x} q \\
& =\Gamma^{-1}(1+\alpha)\left(F_{x}^{(\alpha)}(d x)^{\alpha}+F_{p} d_{x}^{\alpha} p+F_{q} d_{x}^{\alpha} q\right) \\
& =\Gamma^{-1}(1+\alpha)\left(F_{x}^{(\alpha)}+F_{p} p_{x}^{(\alpha)}+F_{q} q_{x}^{(\alpha)}\right)(d x)^{\alpha} .
\end{aligned}
$$

We then have the following set of equations, 


$$
\begin{gathered}
F_{x}^{(\alpha)}+F_{p} p_{x}^{(\alpha)}+F_{q} q_{x}^{(\alpha)}=0, \\
F_{y}+F_{p} p_{y}+F_{q} q_{y}=0, \\
F_{u}+F_{p} p_{u}+F_{q} q_{u}=0,
\end{gathered}
$$

and

$$
\begin{aligned}
G_{x}^{(\alpha)}+G_{p} p_{x}^{(\alpha)}+G_{q} q_{x}^{(\alpha)} & =0, \\
G_{y}+G_{p} p_{y}+G_{q} q_{y} & =0, \\
G_{u}+G_{p} p_{u}+G_{q} q_{u} & =0 .
\end{aligned}
$$

These equations (6.5) to (6.10) provides

$$
\begin{gathered}
\left(F_{p} G_{q}-F_{q} G_{p}\right) p_{y}=F_{q} G_{y}-F_{y} G_{q}, \\
\left(F_{p} G_{q}-F_{q} G_{p}\right) q_{x}^{(\alpha)}=F_{x}^{(\alpha)} G_{p}-F_{p} G_{x}^{(\alpha)}, \\
\left(F_{p} G_{q}-F_{q} G_{p}\right) p_{u}=F_{q} G_{u}-F_{u} G_{p}, \\
\left(F_{p} G_{q}-F_{q} G_{p}\right) q_{u}=F_{u} G_{p}-F_{p} G_{u} .
\end{gathered}
$$

and on substituting into (6.4) we have the fractional PDE

$$
F_{p} G_{x}^{(\alpha)}+F_{q} G_{y}+\left(p F_{p}+q F_{q}\right) G_{u}-\left(F_{x}^{(\alpha)}+p F_{u}\right) G_{p}-\left(F_{y}+q F_{u}\right) G_{q}=0 .
$$

The auxiliary system associated with this equation (see the Appendix) is exactly (6.20).

\section{Example}

Let us consider the equation

$$
p^{2}+q^{2}-(x+y)=0 .
$$

Its solution can be obtained directly by re-writing (6.16) in the form

therefore

$$
p^{2}-x=y-q^{2}=\beta
$$

$$
d u=\Gamma^{-1}(1+\alpha) \sqrt{x+\beta}(d x)^{\alpha}+\sqrt{y-\beta} d y .
$$

Let us use now the Lagrange's auxiliary system (6.2). We then have

$$
\frac{(d x)^{\alpha}}{2 p}=\frac{d^{\alpha} y}{2 q}=\frac{d^{\alpha} u}{2\left(p^{2}+q^{2}\right)}=-\frac{d^{\alpha} p}{-d^{\alpha} x / d x^{\alpha}}=-\frac{d^{\alpha} q}{-1} .
$$

therefore we obtain the two equations

Integrating (6.19) yields

$$
\begin{aligned}
& 2 p\left(d^{\alpha} p\right)=d^{\alpha} x, \\
& 2 q\left(d^{\alpha} q\right)=d^{\alpha} y .
\end{aligned}
$$

$$
\int 2 p p_{x}^{(\alpha)}(d x)^{\alpha}=\int x^{(\alpha)}(d x)^{\alpha}
$$

therefore

In a like manner, one has

$$
p=x+\text { const } .
$$

$$
q=y+\text { const }
$$

and on substituting into (6.16), we still obtain (6.17). 
In the following we shall use these results about FPDE to solve some problems of fractional optimal control.

\section{Variational Approach to Fractional Optimal Control. Model I}

\subsection{Preliminary remarks}

Historically, the optimal control of fractional dynamics appeared in stochastic systems driven by fractional Brownian motion $[8,14]$, and probably for the first time, in mathematical finance, when Mandelbrot pointed out that fractional Brownian motion is much more relevant than the usual one in stock market. We have shown that in some instances (such as portfolio management [21] and optimal control of linear systems with quadratic cost [25]) the stochastic optimal control problem can be converted into a non-random fractional optimal control one by considering the dynamical equations of the state moments of the stochastic system.

As a matter of fact there are several possible models of fractional optimal control problems, and, in a preliminary study, we can more especially mention the following ones:

(i) fractional dynamics with fractional cost function,

$$
S\left(d t^{\alpha}\right), C\left(d t^{\alpha}\right):\left\{\begin{array}{l}
d x=f(x, u, t)(d t)^{\alpha}, \quad 0<\alpha<1, \quad x \in \mathfrak{R} \\
\min _{u} \int_{0}^{T} g(x, u, \tau)(d \tau)^{\alpha}
\end{array}\right.
$$

(ii) fractional dynamics with nonfractional cost function,

$$
S\left(d t^{\alpha}\right), C(d t):\left\{\begin{array}{l}
d x=f(x, u, t)(d t)^{\alpha}, \quad 0<\alpha<1, \quad x \in \mathfrak{R} \\
\min _{u} \int_{0}^{T} g(x, u, \tau) d \tau
\end{array}\right.
$$

(iii) mixed fractional-nonfractional dynamics with fractional cost,

$$
S\left(d t^{\alpha}, d t\right), C\left(d t^{\alpha}\right):\left\{\begin{array}{l}
d x=f_{1}(x, y, u, t) d t, \quad x \in \mathfrak{R}, y \in \mathfrak{R} \\
d y=f_{2}(x, y, u, t)(d t)^{\alpha}, \quad 0<\alpha<1 \\
\min _{u} \int_{0}^{T} g(x, y, u, \tau)(d \tau)^{\alpha}
\end{array}\right.
$$

Here we shall focus mainly on the first system, on which we shall make various hypotheses regarding the differentiabilities of the various functions so involved. Moreover, we point out that the first system and the second one are more or less equivalent as a result of the equality (4.33) which expresses integral w.r.t. $(d t)^{\alpha}$.

\subsection{Necessary optimality conditions for fractional dynamics}

\section{Definition of the Problem}

We consider the one-dimensional system defined by the fractional differential equation

$$
x^{(\alpha)}(t)=f(x, u, t), \quad x(0)=x_{0}, \quad 0<\alpha<1, \quad x \in \mathfrak{R},
$$

with the cost function

$$
G(x(0), u)=h(x(T), T)+\int_{0}^{T} g(x, u, \tau)(d \tau)^{\alpha},
$$

where the integral is taken in the sense of subsection 4.3, and $T$ has a given fixed value. The problem is to determine the optimal control $u^{*}$ which minimizes $G$. We make the following assumption:

(A1) The functions $f():. \mathfrak{R}^{2} \times \mathfrak{R}^{+} \rightarrow \mathfrak{R}, g():. \mathfrak{R}^{2} \times \mathfrak{R}^{+} \rightarrow \mathfrak{R}$ and $h():. \mathfrak{R}^{2} \times \mathfrak{R}^{+} \rightarrow \mathfrak{R}$, all of them are differentiable functions.

Necessary conditions for optimality. 
Define the Hamiltonian $H($.$) by the expression$

$$
H(x, u, p, t):=g(x, u, t)+p(t) f(x, u, t),
$$

where $p(t)$ is the Lagrange parameter (conjugate parameter) of the problem. Then the optimal control is defined by the following equations, referred to as Euler-Lagrange equations.

(i) the dynamical equation (7.1) of the system,

(ii) the necessary optimality condition

$$
H_{u}^{\prime}=0
$$

(iii) the conjugate equation

$$
p^{(\alpha)}(t)=-H_{x}^{\prime}, \quad p(T)=\Gamma^{-1}(1+\alpha) h_{x}^{\prime}(x(T), T) .
$$

Derivation of these equations

As usual we introduce the augmented cost function

$$
\begin{aligned}
G_{a}= & h(x(T), T)+\int_{0}^{T}\left[g+p\left(f-x^{(\alpha)}\right)\right](d \tau)^{\alpha} \\
& =h(x(T), T)+\int_{0}^{T}\left(H-p x^{(\alpha)}\right)(d \tau)^{\alpha} .
\end{aligned}
$$

For a given variation $\delta u$, the corresponding increment $\delta G_{a}$ of $G_{a}$ is

$$
\delta G_{a}=h_{x}^{\prime}(x(T), T) \delta x(T)+\int_{0}^{T}\left[H_{u}^{\prime} \delta u+H_{x}^{\prime} \delta x-p(\delta x)^{(\alpha)}\right](d \tau)^{\alpha},
$$

and on taking account of (4.12), we find that

$$
p(\delta x)^{(\alpha)}=(p \delta x)^{(\alpha)}-p^{(\alpha)} \delta x,
$$

therefore

$$
\delta G_{a}=h_{x}^{\prime}(x(T), T) \delta x(T)+\int_{0}^{T}\left[H_{u}^{\prime} \delta u+\left(H_{x}^{\prime}+p^{(\alpha)}\right) \delta x-(p \delta x)^{(\alpha)}\right](d \tau)^{\alpha} .
$$

We use the general relation

$$
\begin{aligned}
\int\left(y^{(\alpha)}(\tau)\right)(d \tau)^{\alpha} & =\int d^{\alpha} y(\tau), \\
& =\alpha ! \int d y \\
& =\alpha ! y
\end{aligned}
$$

and on inserting into (7.7) we obtain

$$
\begin{aligned}
\delta G_{a} & =h_{x}^{\prime}(x(T), T) \delta x(T)-[\alpha ! p \delta x]_{0}^{T} \\
& +\int_{0}^{T}\left[H_{u}^{\prime} \delta u+\left(H_{x}^{\prime}+p^{(\alpha)}\right) \delta x\right](d \tau)^{\alpha}
\end{aligned}
$$

Equating $\delta G_{a}$ to zero yields the result.

\subsection{Fractional Hamilton-Jacobi equation}

We once more consider the preceding optimal control problem, but here, we use an approach via dynamic programming. Let $G^{*}(x(t), t)$ denote the optimal cost on the interval $(t, T)$. Then the basic optimal equation of dynamic programming reads

$$
\begin{aligned}
G^{*}(x, t) & =\min _{u}\left\{\int_{t}^{t+\delta t} g(x, u, \tau)(d \tau)^{\alpha}+G^{*}(x+\delta x, t+\delta t)\right\} \\
& =\min _{u}\left\{g(x, u, t)(\delta t)^{\alpha}+G^{*}(x, t)+\delta G^{*}\right\},
\end{aligned}
$$

therefore, (as $G^{*}(x, t)$ does not depend upon $\left.u\right)$,

$$
0=\min _{u}\left\{g(x, u, t)(\delta t)^{\alpha}+\delta G *(x(t), t)\right\}
$$

and on making $\delta t \downarrow 0$, 


$$
0=\min _{u}\left\{g(x, u, t)+\frac{1}{\alpha !} \frac{d^{\alpha} G^{*}(x(t), t)}{d t^{\alpha}}\right\} .
$$

This being the case, one has successively

therefore

$$
\begin{aligned}
\left.d G^{*}(x(t), t)\right) & =\frac{\partial G^{*}}{\partial x} d x+\frac{1}{\alpha !} \frac{\partial^{\alpha} G^{*}}{\partial t^{\alpha}}(d t)^{\alpha} \\
& =\frac{1}{\alpha !} \frac{\partial G^{*}}{\partial x} d^{\alpha} x+\frac{1}{\alpha !} \frac{\partial^{\alpha} G^{*}}{\partial t^{\alpha}}(d t)^{\alpha}
\end{aligned}
$$

and

$$
\left.d^{\alpha} G^{*}(x(t), t)\right)=\frac{\partial G^{*}}{\partial x} d^{\alpha} x+\frac{\partial^{\alpha} G^{*}}{\partial t^{\alpha}}(d t)^{\alpha}
$$

$$
\frac{d^{\alpha} G^{*}}{d t^{\alpha}}=\frac{\partial G^{*}}{\partial x} x^{(\alpha)}(t)+\frac{\partial^{\alpha} G^{*}}{\partial t^{\alpha}} .
$$

On substituting this result into (7.10), we obtain the fractional Hamilton-Jacobi equation

$$
g(x, u, t)+f(x, u, t) \frac{\partial G^{*}}{\partial x}+\frac{\partial^{\alpha} G^{*}}{\partial t^{\alpha}}=0 .
$$

\subsection{Lagrangian equations via Hamilton-Jacobi fractional PDE}

In the theory of optimal control, it is well known that the Hamilton-Jacobi equation provides the equations of the Lagrangian approach, and we shall show that this property still holds in the present fractal framework (fortunately!). To this end we shall apply the result of Section 6.

Application to the derivation of the Lagrangian equations

We refer to the equation (7.12) and we define

$$
p:=\frac{\partial G^{*}}{\partial x}, \quad q:=\frac{\partial^{\alpha} G^{*}}{\partial t^{\alpha}},
$$

in such a manner that we can re-write it in the form

or again

$$
f+p f+q=0
$$

$$
H(x, y, u, p)+q=0 .
$$

The auxiliary system (6.2) here reads (with $x \leftarrow t, y \leftarrow x, u^{*} \leftarrow G^{*}, p \leftarrow q, q \leftarrow p$ )

$$
\frac{(d t)^{\alpha}}{F_{q}}=\frac{d^{\alpha} x}{F_{p}}=\frac{d^{\alpha} G^{*}}{p F_{p}+q F_{q}}=-\frac{d^{\alpha} q}{F_{t}^{(\alpha)}+q F_{G^{*}}}=-\frac{d^{\alpha} p}{F_{x}+p F_{G^{*}}} .
$$

Taking the first and last terms, we have

$$
\frac{d^{\alpha} p}{d t^{\alpha}}=-\frac{F_{x}+p F_{G^{*}}}{F_{q}}
$$

with

$$
F_{x}=H_{x}^{\prime}, \quad F_{G^{*}}=0, \quad F_{q}=1
$$

therefore the equation

$$
p^{(\alpha)}(t)=-H_{x}^{\prime}
$$

which is exactly the equation (7.5).

7.4 Extension to cost function defined by integral with respect to (dt)

We once more consider the dynamics described by the equation (7.1) which we re-write below for the convenience of the reader,

$$
x^{(\alpha)}(t)=f(x, u, t), \quad x(0)=x_{0}, \quad 0<\alpha<1, \quad x \in \mathfrak{R},
$$


but we assume the cost function is in the form

$$
J(x(0), u)=h(x(T), T)+\int_{0}^{T} g(x, u, \tau) d \tau,
$$

This model can be easily converted into the preceding one by noticing that the equation (4.28) provides the equality

$$
\int_{0}^{T} g(x, u, \tau) d \tau=\alpha^{-1} \int_{0}^{T}(T-\tau)^{1-\alpha} g(x, u, \tau)(d \tau)^{\alpha}
$$

in such a manner that we can re-write $J($.$) in the form$

$$
J(x(0), u)=h(x(T), T)+\alpha^{-1} \int_{0}^{T}(T-\tau)^{1-\alpha} g(x, u, \tau)(d \tau)^{\alpha},
$$

The Hamiltonian now reads

$$
H(x, u, p, t)=\alpha^{-1}(T-t)^{1-\alpha} g(x, u, t)+p(t) f(x, u, t),
$$

which allows us to duplicate the equations (7.4) and (7.5). Moreover, the Hamiltonian-Jacobi equation (7.12) turns to be

$$
\alpha^{-1}(T-t)^{1-\alpha} g(x, u, t)+f(x, u, t) \partial_{x} J^{*}+\partial_{t}^{\alpha} J^{*}=0 .
$$

\section{Variational Approach to Fractional Optimal Control. Model II 8.1 Necessary optimality conditions}

Definition of the problem

We once more consider the dynamics (7.1) and the cost function (7.2), but now we assume that

(A2) the functions $f(),. g($.$) and h($.$) are \alpha$-th differentiable functions.

Necessary conditions for optimality

With the Hamiltonian $H($.$) still defined by the expression (7.3), the optimal control is$ provided by the following equations:

(i) the dynamical equation (7.1) of the system,

(ii) the necessary optimality conditions

$$
H_{u}^{(\alpha)}(x, u, p, t)=0
$$

(iii) the conjugate equation

$$
p^{(\alpha)}(t)=-\Gamma(2-\alpha) x^{\alpha-1} H_{x}^{(\alpha)},
$$

with the terminal condition

$$
p(T)=\frac{\Gamma(2-\alpha)}{\Gamma(1+\alpha)} x^{\alpha-1}(T) h_{x}^{(\alpha)}(x(T), T) .
$$

Proof

We still refer to the augmented cost which we recall for convenience

$$
G_{a}=h(x(T), T)+\int_{0}^{T}\left(H-p x^{(\alpha)}\right)(d \tau)^{\alpha},
$$

and here, for a given variation $\delta u$, we have the increment of order $\alpha$, say $\delta^{\alpha} G_{a}$,

$$
\begin{gathered}
\delta^{\alpha} G_{\alpha}=h_{x}^{(\alpha)}(x(T), T)(\delta x(T))^{\alpha}+ \\
+\int_{0}^{T}\left(H_{u}^{(\alpha)}(\delta u)^{\alpha}+H_{x}^{(\alpha)}(\delta x)^{\alpha}-p(t) \delta^{\alpha} x^{(\alpha)}\right)(d \tau)^{\alpha} .
\end{gathered}
$$

This being the case, one has

$$
\delta^{\alpha} x^{(\alpha)}=\left(\delta^{\alpha} x\right)^{(\alpha)}=D_{x}^{\alpha}\left(\frac{x^{1-\alpha}}{(1-\alpha) !}(\delta x)^{\alpha}\right),
$$

and on substituting into (8.5) we obtain 


$$
\begin{gathered}
\delta^{\alpha} G_{\alpha}=h_{x}^{(\alpha)}(x(T), T)(\delta x(T))^{\alpha}+ \\
+\int_{0}^{T}\left(H_{u}^{(\alpha)}(\delta u)^{\alpha}+H_{x}^{(\alpha)}(\delta x)^{\alpha}-p(t) D_{x}^{\alpha}\left(\frac{x^{1-\alpha}}{(1-\alpha) !}(\delta x)^{\alpha}\right)\right)(d \tau)^{\alpha},
\end{gathered}
$$

By using (4.11) and (7.8) applied to the $p$-term, we have the equality

$$
\alpha !\left[p \frac{x^{1-\alpha}}{(1-\alpha) !}(\delta x)^{\alpha}\right]_{0}^{T}=\int_{0}^{T} p^{(\alpha)}\left(\frac{x^{1-\alpha}}{(1-\alpha) !}(\delta x)^{\alpha}\right)(d \tau)^{\alpha}+\int_{0}^{T} p D_{x}^{\alpha}\left(\frac{x^{1-\alpha}}{(1-\alpha) !}(\delta x)^{\alpha}\right)(d \tau)^{\alpha},
$$

and on substituting into (8.6), we obtain the increment

$$
\begin{gathered}
\delta^{\alpha} G_{\alpha}=h_{x}^{(\alpha)}(x(T), T)(\delta x(T))^{\alpha} \\
+\int_{0}^{T}\left(H_{u}^{(\alpha)}(\delta u)^{\alpha}+\left(H_{x}^{(\alpha)}+\frac{x^{1-\alpha}}{(1-\alpha) !} p^{(\alpha)}\right)(\delta x)^{\alpha}-D_{x}^{\alpha}\left(p \frac{x^{1-\alpha}}{(1-\alpha) !}(\delta x)^{\alpha}\right)\right)(d \tau)^{\alpha},
\end{gathered}
$$

which can be re-written in the form

$$
\begin{gathered}
\delta^{\alpha} G_{a}=h_{x}^{(\alpha)}(x(T), T)(\delta x(T))^{\alpha}-\left[\alpha ! p \frac{x^{1-\alpha}}{(1-\alpha) !}(\delta x(T))^{\alpha}\right]_{0}^{T} \\
+\int_{0}^{T}\left(H_{u}^{(\alpha)}(\delta u)^{\alpha}+\left(H_{x}^{(\alpha)}+\frac{x^{1-\alpha}}{(1-\alpha) !} p^{(\alpha)}\right)(\delta x)^{\alpha}\right)(d \tau)^{\alpha},
\end{gathered}
$$

Equating $\delta^{a} G_{a}$ to zero yields the result.

\subsection{Optimal control with nondifferentiable constraints}

We once more consider the basic model defined by the dynamics (7.1) and the cost function (7.2), but in addition, we assume that $x(t)$ is restricted to satisfy the condition

$$
F(x)=0 \text {. }
$$

We make the following assumption

(A3) The functions $f, g$ and $h$ all of them are differentiable whilst $F(x)$ is $\alpha$-th differentiable, $0<\alpha<1$.

Introducing the Lagrange parameter $\lambda(t)$ associated with the constraint (8.8), we define the Hamiltonian

$$
H(x, u, p, \lambda, t):=H_{r}(x, u, p, t)+\lambda F(x),
$$

where $H_{r}(x, u, p, t)$ is the Hamiltonian (7.3) of the (free) system without the constraint (8.8).

Due to the $\alpha$-th differentiability of $F(x)$, we can set the problem in the framework of the subsection 8.1 above, and we shall have the optimality equations

$$
\begin{gathered}
\partial_{u}^{\alpha} H_{r}(x, u, p, t)=0, \\
p^{(\alpha)}(t)=-\Gamma(2-\alpha) x^{\alpha-1}\left(\partial_{x}^{\alpha} H_{r}+\lambda F^{(\alpha)}\right),
\end{gathered}
$$

with the equations (7.1) and (8.8) and the terminal condition (8.3).

\section{Application to Fractional Analytical Mechanics}

\section{Fractional canonical equations}

First of all, the above equations can be generalized in a straightforward manner to deal with vector state $x \in \mathfrak{R}^{m}$. We shall have $m$ equations (7.1) each one indexed by the subscript $i \in\{1, . ., m\}$.

This being the case, as it is customary in physics, one can explicitly introduce the freedom degree of the system by expressing $x$ in terms of $n$ generalized co-ordinates $q_{1}, q_{n}, . ., q_{n}$ and write $x=x\left(q_{1}, q_{2}, \ldots, q_{n}, t\right)$, in which case the canonical equation turn to be 


$$
\begin{aligned}
q_{i}^{(\alpha)}(t) & =\partial_{p_{i}} H, \\
p_{i}^{(\alpha)}(t) & =-\partial_{q_{i}} H .
\end{aligned}
$$

Fractional velocity

We shall assume that there is some coarse graining phenomenon which causes that the trajectory $q(t)$ exhibits some roughness. As a result, the increment of the displacement is not $d q=o(d t)$, but rather is $d q=o\left((d t)^{\alpha}\right), 0<\alpha<1$. In this framework, in quite a natural way, we shall refer to the quotient $d q /(d t)^{\alpha}$, which we shall convert into the fractional velocity $u$,

$$
u=\frac{d^{\alpha} q}{(d t)^{\alpha}}
$$

by using the equation (4.15) which relates $d^{\alpha} q$ and $d q$.

Action function

We shall assume that the system is still defined by its Lagrangian function $L\left(q, q^{(\alpha)}, t\right)$ and that its dynamics evolves in such a way to minimizes the integral, referred to as action integral,

$$
S=\int_{t_{1}}^{t_{2}} L\left(q, q^{(\alpha)}, \tau\right)(d \tau)^{\alpha},
$$

for any arbitrary interval $\left[t_{1}, t_{2}\right]$. The Lagrangian itself is a straightforward generalization of the classical definition which, in the case when we restrict ourselves to Newton mechanics, will be selected in the form

$$
L\left(q, q^{(\alpha)}, t\right)=K\left(q, q^{(\alpha)}, t\right)-V(q),
$$

where $K$ is the kinetic energy which can be written in the form

$$
K=\frac{1}{2} \sum_{i j} q_{i}^{(\alpha)} q_{j}^{(\alpha)},
$$

and $V(q)$ denote the potential energy function of the system, which is now defined by the dynamical vector equation

$$
q^{(\alpha)}(t)=u .
$$

Shortly, the fractional derivative $q^{(\alpha)}(t)$ is substituted for $\dot{q}(t)$ in the classical equations.

Conservative mechanical system

According to the Hamiltonian principle, the trajectory from 0 to any $T$ is such that the action $S$ has a stationary value. This being the case, the Hamiltonian is

$$
H=L+p^{T} u,
$$

where the superscript $T$ denotes the transpose, whereby we obtain the Euler-Lagrange conditions

$$
\begin{aligned}
D^{\alpha} p^{T} & =-\partial_{q} H=-\partial_{q} L, \\
\partial_{u} H & =\partial_{u} L+p^{T}=0 .
\end{aligned}
$$

On combining these two (vector) equations, we obtain

$$
\frac{\partial L}{\partial q}-\frac{d^{\alpha}}{d t^{\alpha}}\left(\frac{\partial L}{\partial q^{(\alpha)}}\right)=0 .
$$

Nonconservative system

In the case when the mechanical system is subject to an external force $Q$, the trajectory of the system is such that

$$
\delta \int_{0}^{T} L(q, u, t)(d t)^{\alpha}+\int_{0}^{T} Q^{T}(q) \delta q(d t)^{\alpha}=0
$$


and expanding the calculation yields

$$
\frac{\partial L}{\partial q}-\frac{d^{\alpha}}{d t^{\alpha}}\left(\frac{\partial L}{\partial q^{(\alpha)}}\right)+Q=0
$$

Illustrative example

Let us assume that the system is a particle of mass $m$ subject to the potential $V(q)=-\rho q^{2} / 2$, where $\rho$ denotes a positive constant. The Lagrangian $L:=T-V$ is then $L=(1 / 2)\left(m u^{2}+\rho q^{2}\right)$, and $(9.11)$ provides the equation

$$
m q^{(2 \alpha)}(t)=-\rho q .
$$

Assume that $0<\alpha \leq 1 / 2$, then the equation (9.14) yields

$$
q(t)=q_{0} E_{2 \alpha}\left(-(\rho / m) t^{2 \alpha}\right)
$$

Assume now that $1 / 2<\alpha \leq 1$, then one has

$$
\begin{aligned}
q(t) & =C_{1} E_{\alpha}\left(i \sqrt{\frac{\rho}{m}} t^{\alpha}\right)+C_{2} E_{\alpha}\left(-i \sqrt{\frac{\rho}{m}} t^{\alpha}\right) \\
& =\widetilde{C}_{1} \cos _{\alpha}\left(\sqrt{\frac{\rho}{m}} t^{\alpha}\right)+\widetilde{C}_{2} \sin _{\alpha}\left(\sqrt{\frac{\rho}{m}} t^{\alpha}\right),
\end{aligned}
$$

with the definition

$$
E_{\alpha}(i t)=\cos _{\alpha} t+i \sin _{\alpha} t .
$$

In the special case when $q(0)=q_{0}$ and $q^{(\alpha)}(0)=0$, we finally obtain

$$
q(t)=q_{0} \cos _{\alpha}\left(\sqrt{\frac{\rho}{m}} t^{\alpha}\right) .
$$

\section{Mechanical Systems with Nondifferentiable Constraints}

Here, we directly refer to the results of the section 8 .

Let us consider a one-dimensional system. With the notation of Section 9, the Hamiltonian now reads

$$
H=L\left(q, q^{(\alpha)}, t\right)+p u+\lambda F(q),
$$

and the equations (8.10) and (8.11) yield successively

$$
\frac{\partial^{\alpha} L}{\partial u^{\alpha}}+\frac{1}{(1-\alpha) !} p u^{1-\alpha}=0
$$

and

$$
\frac{d^{\alpha} p}{d t^{\alpha}}=-\frac{(1-\alpha) !}{q^{1-\alpha}}\left(\frac{\partial^{\alpha} L}{\partial q^{\alpha}}+\lambda \frac{\partial^{\alpha} F}{\partial q^{\alpha}}\right) .
$$

Taking $p^{(\alpha)}$ from (10.2) and equating to (10.3) yields the equation (on assuming the presence of an external force $Q$ for the sake of generalization),

with

$$
\frac{(1-\alpha) !}{q^{1-\alpha}}\left(\frac{\partial^{\alpha} L}{\partial q^{\alpha}}+\lambda \frac{\partial^{\alpha} F}{\partial q^{\alpha}}+Q\right)-(1-\alpha) ! \frac{d^{\alpha}}{d t^{\alpha}}\left(u^{\alpha-1} \frac{\partial^{\alpha} L}{\partial u^{\alpha}}\right)=0 .
$$

$$
q^{(\alpha)}(t)=u
$$

Illustrative example

We once more consider a particle of mass $m$ subject to the external force $Q$ and subject to the condition $F(q)=0$. Its Lagrangian is $L=(1 / 2) m u^{2}$, and (10.4) direct yields the equation 


$$
\frac{(1-\alpha) !}{q^{1-\alpha}}\left(\lambda \frac{\partial^{\alpha} F}{\partial q^{\alpha}}+Q\right)-\frac{(1-\alpha) !}{(2-\alpha) !} m q^{(2 \alpha)}=0
$$

or again

$$
m q^{(2 \alpha)}(t)=(2-\alpha) ! q^{\alpha-1}\left(Q+\lambda \partial_{q}^{\alpha} F\right)
$$

Let us consider the special approximation in which the coefficient $\left(Q+\lambda \partial_{q}^{\alpha} F\right)$ is a constant, in such a manner that (10.7) can be shortened in the form

$$
q^{(2 \alpha)}(t)=K(\alpha) q^{\alpha-1}
$$

and we shall have two different families of solutions depending upon the value of $\alpha$,

First, assume that $2 \alpha \leq 1$. Then we re-write (10.8) in the form

$$
(2-\alpha) q^{1-\alpha} q^{(2 \alpha)}=(2-\alpha) K(\alpha)
$$

and we recognize that the left side is the $2 \alpha$-th derivative of $q^{2-\alpha}$ (see the chain rule of fractional derivative in (4.12)), in such a manner that we have the equation

$$
D_{t}^{2 \alpha} q^{2-\alpha}=m^{-1}((2-\alpha) !)^{2} K(\alpha)
$$

of which the solution is

$$
q^{2-\alpha}=\frac{2-\alpha}{(2 \alpha) !} K(\alpha) t^{2 \alpha} .
$$

Assume now that $1<2 \alpha<2$. In this case, we shall set $2 \alpha=: 1+\beta, 0<\beta<1$, to re-write (10.8) in the form

$$
q^{(1+\beta)}=K(\alpha) q^{(\beta-1) / 2}, \quad \alpha=(1+\beta) / 2 .
$$

This being the case, the solutions for $\beta=0$ on the one hand, and for $\beta=1$ on the other hand suggest to look for a solution in the form $q=C t^{\gamma}$, and on substituting into (10.10) we find

$$
q(t)=C t^{\gamma} \quad \text { with } \quad \gamma:=\frac{2 \beta+2}{3-\beta}
$$

and

$$
C=\left(K(\alpha) \frac{\Gamma(\gamma-\beta+1}{(\gamma-\beta) \Gamma(\gamma+1)}\right)^{\gamma /(\gamma-\beta+1)} .
$$

Remark that when $\beta=0$, that is to say when $\alpha=1 / 2$, in (10.11), one comes across (10.9). In other words, there is continuity of the solution with respect to $\alpha$.

\section{Why do We Need Complex Variables and Brownian Motion? \\ 11.1 Introduction to the modeling problem}

In the present section, given this fractional calculus framework, we shall examine whether we need to use complex variables and/or the Brownian motion to refine the model. The key of our reflection is Bohr's correspondence principle in accordance of which the laws of quantum mechanics are such that, when the considered mechanical systems involve a large number of quanta, then one should come across the classical equations. In our approach, to comply with this principle, the first idea is to try using a non-random model in the form

$$
d x=f_{1}(x, t) d t+f_{2}(x, t)(d t)^{\alpha}, \quad 0<\alpha<1,
$$

and the question of course is as to whether this model is meaningful. Unfortunately, the answer is no, unless $\alpha=1 / 2$. 
Indeed, assume that $\alpha=1 / n, n$ positive integer. According to the fractional Taylor series, one can write

$$
d x=\sum_{k=2}^{n-1} \frac{1}{(\alpha k) !} x^{(\alpha k)}(d t)^{\alpha k}+\frac{1}{\alpha !} x^{(\alpha)}(d t)^{\alpha}+\dot{x} d t,
$$

therefore, on comparing with (11.1), we should have the identities

$$
\begin{gathered}
\dot{x}(t)=f_{1}(x, t), \\
x^{(\alpha)}(t)=\alpha ! f_{2}(x, t), \\
\sum_{k=2}^{n-1} \frac{1}{(\alpha k) !} x^{(\alpha k)}(d t)^{\alpha k}=0,
\end{gathered}
$$

which provide the necessary conditions

$$
f_{1}(x, t)=\alpha ! D_{t}^{1-\alpha} f_{2}(x, t),
$$

and

$$
x^{(\alpha k)}(t)=0, \quad k=2, \ldots, n-1 .
$$

But the condition $x^{(\alpha)}(t)=0$ implies that $x^{(k \alpha)}(t)=0$ for every $k>2$, with our fractional derivative definition, and thus $x^{(n \alpha)}(t)=\dot{x}(t)=0$. The only case when the equation (11.1) is meaningful is $\alpha=1 / 2$, to yield the dynamics

$$
d x=\dot{x}(t) d t+\Gamma^{-1}(3 / 2) x^{(1 / 2)}(d t)^{1 / 2},
$$

therefore the consistency condition

$$
f_{1}(x, t)=\Gamma(3 / 2) D_{t}^{(1 / 2)} f_{2}(x, t) .
$$

But even this equation (11.8) is not quite satisfactory since then we can drop the term $\dot{x} d t$ and write

$$
d x=\Gamma^{-1}(3 / 2) x^{(1 / 2)}(d t)^{1 / 2}+o(d t)
$$

The conclusion is that $f_{1} d t$ and $f_{2}(d t)^{\alpha}$ in (11.1) are of different physical natures, and in order to take account of this feature, we can either set the problem in the complex plane or describe the nondifferentiability of the variables by using stochastic noises, or combine these two approaches.

\subsection{Strip modeling of mechanical systems}

Complex variable as $s$ result of irreversibility of time

Many readers are not fully convinced by the Nottale's expression (2.5) of complex velocity, and incidentally here we shall see that the formalism of fractional Taylor's series can contribute some arguments to support this complex plane approach.

Indeed, strictly speaking, the expression (3.3) of the fractional derivative holds for positive $h$ only, clearly, one should write

$$
d x_{+}(t)=(\alpha !)^{-1} x_{+}^{(\alpha)}(t)(d t)^{\alpha}, \quad d t>0 .
$$

In other words, for negative $d t$, in quite a natural way, we are entitled to write the equality

$$
d x_{-}(t)=-i^{2 \alpha}(\alpha !)^{-1} x_{-}^{(\alpha)}(t)(d t)^{\alpha}, \quad d t<0 .
$$

where $x_{-}^{(\alpha)}$ would be the $\alpha$-th derivative on the left. Clearly, complex variables appear in quite a natural way as a direct result of the presence of the term $(d t)^{\alpha}$ which pictures the irreversibility of time.

We then have the two equations

$$
\begin{gathered}
d x_{+}=v_{+} d t+u_{+}(d t)^{\alpha}, \quad d t>0, \\
d x_{-}=v_{-} d t-i^{2 \alpha} u_{-}(d t)^{\alpha}, \quad d t<0,
\end{gathered}
$$


where $x_{+}, x_{-}, v_{+}, v_{-}, u_{+}$and $u_{-}$all are real valued variables. Once again, we bear in mind that the presence of $i$ in the equation (11.11) is due to the irreversibility of time. These equations are equivalents to the two equations

$$
\begin{aligned}
& d\left(x_{+}+x_{-}\right)=\left(v_{+}+v_{-}\right) d t+\left(u_{+}-i^{2 \alpha} u_{-}\right)(d t)^{\alpha}, \\
& d\left(x_{+}-x_{-}\right)=\left(v_{+}-v_{-}\right) d t+\left(u_{+}+i^{2 \alpha} u_{-}\right)(d t)^{\alpha} .
\end{aligned}
$$

This being the case, assume now that we want to combine (11.12) and (11.13) in only one equation, the easier way to to do that is to use complex variables, and for instance, on generalizing Nottale's formulation, one can introduce the variables

$$
\begin{aligned}
\tilde{x} & :=2^{-1}\left(x_{+}+x_{-}\right)-i^{2 \alpha} 2^{-1}\left(x_{+}-x_{-}\right), \\
\tilde{v} & :=2^{-1}\left(v_{+}+v_{-}\right)-i^{2 \alpha} 2^{-1}\left(v_{+}-v_{-}\right),
\end{aligned}
$$

to obtain the equation

$$
d \widetilde{x}=\widetilde{v} d t+\left(1-i^{2 \alpha}\right) 2^{-1}\left(u_{+}-u_{-}\right)(d t)^{\alpha} .
$$

Brownian motion and nondifferentiability

In the equation (11.15), $\widetilde{v} d t$ is the differentiable component of the trajectory, whilst the $\left(u_{+}-u_{-}\right)$-term describes its nondifferentiability. This being the case, it is well known that a function which is continuous everywhere but nowhere differentiable cannot be replicated: in other words it exhibits a random-like feature.

A practical useful way to take account of such a random-like nondifferentiability is to use a Brownian motion $w(t)$ with zero mean and the variance $\sigma^{2}$ and to set

$$
d \widetilde{x}(t)=\tilde{v} d t+\left(1-i^{2 \alpha}\right) \sigma w(t)(d t)^{\alpha} .
$$

In the special case when $\alpha=1 / 2$, we have

$$
d \widetilde{x}(t)=\widetilde{v} d t+(1-i) \sigma w(t) \sqrt{d t},
$$

and we so come across the strip modeling and the model of Brownian motion with complex variance which we have introduced previously $[18,19,20]$.

In quite a natural way, this suggests to generalize the modeling in the complex plane in the form [19]

$$
d z(t)=v d t+\sigma w_{n}(t)(d t)^{1 / n}
$$

where $z \in C$ and where $w_{n}(t)$ denotes a complex-valued white noise of order $n \in N-\{0\}$

\section{Introduction to Differential Geometry of Fractional Order}

In the present section, all the notations and parameters are local ones (i.e. for this section only) and mainly we shall use the notation of tensor calculus regarding the indexes of co-variant and contra-variant co-ordinates.

Given this prerequisite, it is easy to see that the fractional calculus of the preceding section provide an easy way to extend some results of elementary differential geometry.

As it is customary, we consider a generic point $M$ defined by the rectilinear co-ordinates $x^{j}, j=1,2, \ldots, m$

$$
x^{j}=f^{j}\left(u^{1}, u^{2}, \ldots, u^{n}\right)=f^{j}(u),
$$

where $u^{i}, i=1, \ldots, n$ denote the curvilinear coordinates. Using Einstein's summation convention, the natural basis $\left(\vec{e}_{1}, \ldots, \vec{e}_{n}\right)$ is defined by the relation

$$
\begin{aligned}
d^{\alpha} \vec{M} & =\vec{e}_{i}\left(d u^{i}\right)^{\alpha} \\
& =\partial_{i}^{\alpha} \vec{M}\left(d u^{i}\right)^{\alpha},
\end{aligned}
$$


with $\partial_{i}^{\alpha} \equiv \partial^{\alpha} /\left(\partial u^{i}\right)^{\alpha}$.On generalizing the so-called Christoffel coefficients $\Gamma_{j k}^{i}$ in the form $\Theta_{j k}^{i}$, we shall write

therefore

$$
\partial_{k}^{\alpha} \vec{e}_{j}=\widetilde{\Gamma}_{j k}^{i} \vec{e}_{i}
$$

$$
d^{\alpha} \vec{e}_{j}=\widetilde{\Gamma}_{j k}^{i}\left(d u^{k}\right)^{\alpha} \vec{e}_{i}
$$

Introducing the notations (i.e. the counterpart of $\omega_{i}^{j}$ )

$$
\widetilde{\omega}_{j}^{i}=\widetilde{\Gamma}_{j k}^{i}\left(d u^{k}\right)^{\alpha},
$$

which is the counterpart of $\omega_{i}^{j}$ in tensor calculus, we have as well

$$
d^{\alpha} \vec{e}_{j}=\widetilde{\omega}_{j}^{i} \vec{e}_{i} .
$$

Given the vector $\vec{v}=v^{i} \vec{e}_{i}$, the absolute differential $d_{T}^{\alpha} v^{i}$ of its components are defined by the equality

$$
d^{\alpha} \vec{v}=\left(d_{T}^{\alpha} v^{i}\right) e_{i}
$$

and can be obtained by the following equalities

$$
\begin{aligned}
d^{\alpha} \vec{v} & =d^{\alpha}\left(v^{i} \vec{e}_{i}\right) \\
& =\left(d^{\alpha} v^{i}\right) \vec{e}_{i}+v^{i} d^{\alpha} \vec{e}_{i} \\
& =\left(d^{\alpha} v^{i}\right) \vec{e}_{i}+v^{i} \widetilde{\omega}_{i}^{j} \vec{e}_{j} .
\end{aligned}
$$

According to the equation (12.2) we can go a step further and define the $\alpha$-differential

$$
\left(d^{\alpha} s\right)^{2}=\widetilde{g}_{i j}\left(d u^{i}\right)^{\alpha}\left(d u^{j}\right)^{\alpha} \text { with } \widetilde{g}_{i j}:=\vec{e}_{i} \vec{e}_{j},
$$

but on doing so, we explicitely assume that all the differentials $d u^{i}$, for all $i$, can take on positive values only.

By duplicating classical calculus, one can get easily the relations between $\widetilde{\Gamma}_{i j}^{k}$ and $\widetilde{g}_{r h}$. Indeed, one has successively

$$
\begin{gathered}
d^{\alpha} \widetilde{g}_{i j}=\vec{e}_{i} d^{\alpha} \vec{e}_{j}+\vec{e}_{j} d^{\alpha} \vec{e}_{i} \\
=\vec{e}_{i} \widetilde{\omega}_{j}^{k} \vec{e}_{k}+\vec{e}_{j} \widetilde{\omega}_{i}^{k} \vec{e}_{k}
\end{gathered}
$$

therefore the Ricci identities of fractional order

$$
d^{\alpha} \widetilde{g}_{i j}=\widetilde{g}_{i k} \widetilde{\omega}_{j}^{k}+\widetilde{g}_{j k} \widetilde{\omega}_{i}^{k}
$$

and

$$
\partial_{k}^{\alpha} \widetilde{g}_{i j}=\tilde{g}_{i h} \widetilde{\Gamma}_{j k}^{h}+\widetilde{g}_{j h} \widetilde{\Gamma}_{i k}^{h} .
$$

One can go a step further, and define the difference

$$
d^{\alpha} \delta^{\alpha} \vec{e}_{j}-\delta^{\alpha} d^{\alpha} \vec{e}_{j}=\widetilde{\Omega}_{j}^{h} \vec{e}_{h},
$$

with

$$
\widetilde{\Omega}_{j}^{h}=\widetilde{R}_{j, r s}^{h}\left(d u^{r}\right)^{\alpha}\left(d u^{s}\right)^{\alpha},
$$

where $\widetilde{R}_{j, r s}^{h}$ would be the fractional counterpart of the Riemann-Christofel symbol.

One can so expect to expand a Riemannian geometry of fractional order and then to examine whether it may have a meaning in theoretical physics.

\section{Fractals and Special Relativity} 13.1 Guessing for an invariance principle 
Again on assuming that there exists a finite resolution for space and time coordinates due to some kind of "coarse graining", we can try to generalize well known classical statements, and say that the Galilean transformation $(x, y, z, t) \rightarrow\left(x^{\prime}, y^{\prime}, z^{\prime}, t^{\prime}\right)$ of the special relativity of fractional order is such that the relation

$$
\left(d^{\alpha} x\right)^{2}+\left(d^{\alpha} y\right)^{2}+\left(d^{\alpha} z\right)^{2}=\tilde{c}^{2}(d t)^{2 \alpha},
$$

is equivalent to

$$
\left(d^{\alpha} x^{\prime}\right)^{2}+\left(d^{\alpha} y^{\prime}\right)^{2}+\left(d^{\alpha} z^{\prime}\right)^{2}=\widetilde{c}^{2}\left(d t^{\prime}\right)^{2 \alpha},
$$

where $\widetilde{c}$ is a positive constant of which the meaning remains to be identified.

Alternatively, we can follow the very general statement made by Minkowski, and say that the variation of the four-dimensional (squared) "line element"

$$
d s^{2 \alpha}=\widetilde{c}^{2} d t^{2 \alpha}-\left(d^{\alpha} x\right)^{2}-\left(d^{\alpha} y\right)^{2}-\left(d^{\alpha} z\right)^{2}
$$

vanishes in all reference frame:

$$
\delta\left(d s^{2 \alpha}\right)=0
$$

Remarks and comments.

The constant $\widetilde{c}$ has been used here (instead of $c$ ) to ascertain that we do not introduce inconsistency with physics, since we remain in a pure mathematical framework. Nevertheless, following well known rationale, $\widetilde{c}^{2}$ should be the maximum value of the fractional velocity square $v_{\alpha}{ }^{2}$; and the question of course is as to whether one has $\tilde{c}<c$, or $\tilde{c}=c$ or $\tilde{c}>c$.

Remark that the expression (13.3) exhibits a symmetry breaking between time on the one hand, and space co-ordinates on the other hand since its involves both $(d t)^{\alpha}$ and $d^{\alpha} x$, and this might be disturbing at first glance. Let us examine this question more closely.

\subsection{On the Minkowskian pseudo-metric}

Preliminary remark. According to the equation (4.17), the equation (4.17) the $\alpha$-velocity $d^{\alpha} x / d t^{\alpha}$ is related with the velocity $d x / d t$ by the equation

$$
\frac{d^{\alpha} x}{d t^{\alpha}}=\frac{1}{(1-\alpha) !} x^{1-\alpha}\left(\frac{d x}{d t}\right)^{\alpha}, \quad 0<\alpha \leq 1 .
$$

This being the case, the Minkoskian pseudo-metric (with $t$ and $x$ only)

$$
d s^{2}=c^{2} d t^{2}-d x^{2}
$$

starts from the principle in accordance of which one has necessarily $(d x / d t)^{2} \leq c^{2}$ where $c$ denotes the velocity of light (or of electromagnetic waves) in vacuum, and in a like manner this suggests to postulate that this inequality should hold also for the $\alpha$-velocity, clearly

$$
\left(d^{\alpha} x / d t^{\alpha}\right)^{2} \leq c_{\alpha}{ }^{2}
$$

with $c_{\alpha}$ denoting the $\alpha$-velocity of light. According to (13.5), the latter is

$$
c_{\alpha}=\frac{1}{(1-\alpha) !} x^{1-\alpha} c^{\alpha}
$$

but, since one has $x=c t$, it follows that

$$
c_{\alpha}=c \frac{t^{1-\alpha}}{(1-\alpha) !} .
$$

Substituting this value into (13.6) provides the inequality

$$
\left(\frac{d^{\alpha} x}{d t^{\alpha}}\right)^{2} \leq c^{2} \frac{t^{2(1-\alpha)}}{\Gamma^{2}(2-\alpha)}
$$

therefore the pseudo-metric 


$$
\begin{aligned}
(d z)_{1}^{2} & =c^{2} \Gamma^{-2}(2-\alpha) t^{2(1-\alpha)}(d t)^{2 \alpha}-\left(d^{\alpha} x\right)^{2} \\
& =\Gamma^{-2}(2-\alpha)\left(c^{2} t^{2(1-\alpha)}(d t)^{2 \alpha}-\Gamma^{2}(2-\alpha)\left(d^{\alpha} x\right)^{2}\right) .
\end{aligned}
$$

Next, the equation (13.5) yields

$$
(d t)^{\alpha}=(1-\alpha) ! t^{\alpha-1} d^{\alpha} t
$$

and on substituting this expression into (13.9), we have

$$
(d s)_{1}^{2}=\Gamma^{-2}(2-\alpha)\left(c^{2}\left(d^{\alpha} t\right)^{2}-\left(d^{\alpha} x\right)^{2}\right) .
$$

Clearly, we conclude that the $\alpha$-Minkowskian pseudo-metric $d_{\alpha} s$ is defined by the expression

and by virtue of (4.15)

$$
\Gamma^{2}(2-\alpha)\left(d_{\alpha} s\right)^{2}=c^{2}\left(d^{\alpha} t\right)^{2}-\left(d^{\alpha} x\right)^{2},
$$

$$
\left(d_{\alpha} s\right)^{2}=\frac{\Gamma^{2}(1+\alpha)}{\Gamma^{2}(2-\alpha)}(d s)^{2} .
$$

The corresponding Lorentz transformation reads

$$
\begin{gathered}
d^{\alpha} x^{\prime}=\rho\left(v_{\alpha}\right)\left(d^{\alpha} x+v_{\alpha} d^{\alpha} t\right), \\
d^{\alpha} t^{\prime}=\rho_{\alpha}(v)\left(d^{\alpha} t+\frac{v_{\alpha}}{c^{2}} d^{\alpha} x\right),
\end{gathered}
$$

with

$$
\rho\left(v_{\alpha}\right):=\left(1-\frac{v_{\alpha}{ }^{2}}{c^{2}}\right)^{-1 / 2}
$$

where $v_{\alpha}$ denotes a constant.

In other words, the fractional Lagrangian mechanics herein proposed is fully consistent with the special relativity.

\section{Concluding Remarks}

The main contribution of the present article can be summarized as follows.

(i) We now have at hand a fractional calculus ruled by a fractional Taylor's series which is completely parallel to standard calculus. In order to obtain this result, we have had to come back to the definition of fractional derivative, and mainly we have proposed a definition which is based on difference of fractional order, and we have slightly modified the so-called Riemann-Liouville definition to circumvent the problem caused by the initial values of the considered functions.

It is of order to point out the difference between our derivation of fractional Taylor's series and Kolwankar one's. This author considers functions defined on sets which are themselves fractal and thus cause the fractional differentiability of the considered function, whilst the functions which we are dealing with are defined on usual sets. Here, we use fractional derivative as a modeling of variation. In addition, the theoretical framework of our approach is very elementary (first course of calculus!) whilst Kolwantar's derivation is a bit more sophisticated.

(ii) By using this fractional calculus, we have been able to generalize the Lagrange's characteristics technique for solving PDE and to obtain the fractional Hamilton-Jacobi equation of a broad class of nonlinear fractional partial differential equations.

(iii) By this way, we had at hand the necessary prerequisite to consider the optimal control of fractional dynamics with fractional cost, and therefore to switch to fractional Lagrangian mechanics.

(iv) We took this opportunity to outline prospects for a possible Riemannian geometry of fractional order on the one hand, and to examine how our fractal modeling could be introduced in special relativity. 
Basically, there is some breaking symmetry between time and space co-ordinates, and complex state variables appear in quite a natural way as a result of irreversibility of time. In addition, we think that we have contributed some arguments to support the complex velocity introduced by Nottale in its fractal approach.

Some suggestions for further research are the followings:

(i) To get more insight in the analysis of the equilibrium position of a mechanical system using the fractional Taylor's series.

(ii) To expand the section 12 in a Riemmannian geometry of fractional order, and to examine whether the results so obtained might have a meaning in theoretical physics.

(iii) To examine whether the fractional equation

$$
i \hbar \frac{\partial^{\alpha} \psi}{\partial t^{\alpha}}=\hat{H}_{\alpha} \psi
$$

where $\hat{H}_{\alpha}$ is a self-adjoint operator which could possibly depend upon $\alpha$, may have a meaning in quantum mechanics.

(iv) Fractal Brownian motion can be generated by Poissonian noises [32,33], and it could be of interest, for instance in biosciences, to systematically investigate the fractal modeling of some of theses processes [7,41]

(v) Let $w(t)$ denote a Gaussian white noise with zero mean and the variance $\sigma^{2}$. Assume that $f(x)$ has derivatives of order $(1 / 2) k, k$ integer, then one can write

$$
f\left(x+w^{2} d t\right) \cong f(x)+\Gamma^{-1}(3 / 2) w(t) \sqrt{d t}+f_{x}^{\prime}(x) w^{2}(t) d t,
$$

and the expectation with respect to $w$ yields

$$
E_{w}\left\{f\left(x+w^{2} d t\right\} \cong f(x)+f_{x}^{\prime}(x) \sigma^{2} d t .\right.
$$

Consider now the differentiable function $g(x)$; then one has

$$
g(x+w \sqrt{d t}) \cong g(x)+g_{x}^{\prime}(x) w(t) \sqrt{d t}+(1 / 2) g_{x}^{\prime \prime}(x) w^{2}(t) d t,
$$

therefore

$$
E_{w}\{g(x+w \sqrt{d t})\} \cong g(x)+(1 / 2) g_{x}^{\prime \prime}(x) \sigma^{2} d t .
$$

The similarity between the equations (14.2) and (14.3) is striking, and deserves more study, regarding mainly its possible effects in the modeling of physical phenomena. The basic question is as to why should we select one of them instead of the other one?

Remark that if a function is differentiable, it is also $\alpha$-th differentiable, $0<\alpha<1$, in such a manner that we could assume that both $f(x)$ and $g(x)$ are differentiable.

(vi) If we set $\alpha=1 / n, n$ positive integer; are there some values of $n$ which are more especially relevant in physics?

\section{Appendix A1}

Some basic formmlas inferred by fractional Taylor's series

In this appendix we give some formulas which are direct consequences of our fractional Taylor series and which can be of help for further calculation. One assume that $0<\alpha \leq 1$.

$$
D^{\alpha} t^{\gamma}=\frac{\Gamma(\gamma+1)}{\Gamma(\gamma+1-\alpha)} t^{\gamma}
$$




$$
\begin{aligned}
& d^{\alpha} t=\Gamma^{-1}(2-\alpha) t^{1-\alpha}(d t)^{\alpha} \\
& \int_{0}^{t} y(\tau)(d \tau)^{\alpha}=\alpha \int_{0}^{t}(t-\tau)^{\alpha-1} y(\tau) d \tau, \quad 0<\alpha \leq 1 \\
& \int_{0}^{t} y^{(\alpha)}(\tau)(d \tau)^{\alpha}=\int_{0}^{t} d^{\alpha} y=\Gamma(1+\alpha) \int_{0}^{t} d \tau=\Gamma(1+\alpha)\left(t-t_{0}\right) \\
& \int_{0}^{t} d^{\alpha} \tau=t \Gamma(\alpha+1) \\
& y=E_{\alpha}(x) \equiv x=\ln _{\alpha} y \\
& \int_{0}^{t} \frac{d^{\alpha} \tau}{\tau}=\ln _{\alpha} t \\
& E_{\alpha}\left((x+y)^{\alpha}\right)=E_{\alpha}\left(x^{\alpha}\right) E_{\alpha}\left(y^{\alpha}\right) \\
& \left(\ln _{\alpha}(u v)^{\alpha}\right)=\left(\ln { }_{\alpha} u\right)^{1 / \alpha}+(\ln \alpha v)^{1 / \alpha} \\
& \int_{\frac{(d t)^{\alpha}}{t}}=\frac{t^{\alpha-1}-1}{(\alpha-1) \Gamma(2-\alpha)} \\
& \frac{d^{\alpha} y}{d t^{\alpha}}=\frac{1}{\Gamma(2-\alpha)} x^{1-\alpha}\left(\frac{d x}{d t}\right)^{\alpha}
\end{aligned}
$$

\section{Apppendix A2}

\section{$\alpha$-Anti-Derivative of $1 / \mathbf{u}$}

We cannot apply the formula

$$
D^{\alpha} x^{\gamma}=\frac{\Gamma(\gamma+1)}{\Gamma(\gamma+1-\alpha)} x^{\gamma-\alpha}
$$

with $\gamma=1$, and exactly like for the pair (exponential, logarithm) we shall work by means of inverse function.

We consider the pair

and we start with the identity

$$
y=f(x) \quad, \quad x=f^{i n v}(y)=g(y)
$$

$$
y=f(g(y))
$$

of wich the $\alpha$-derivative is

$$
\frac{d^{\alpha} y}{d y^{\alpha}}=\frac{\Gamma(2)}{\Gamma(2-\alpha)} y^{1-\alpha}=\frac{d f}{d g} g^{(\alpha)}(y) .
$$

In the following, we shall determine which function $g(y)$ satisfies the condition

$$
g^{(\alpha)}(y)=1 / y
$$

To this end, on combining (A.3) and (A.4) we have

$$
\frac{d f}{d g}=\frac{y^{2-\alpha}}{\Gamma(2-\alpha)} .
$$

But, according to (A.1) one has $d f=d y$, in such a manner that (A.5) yields

$$
y^{\alpha-2} d y=\Gamma(2-\alpha) d g,
$$

from where we obtain 


$$
g(y)=\frac{y^{\alpha-1}}{(\alpha-1) \Gamma(2-\alpha)}+\text { const } .
$$

For the sake of consistency, we shall select the additive constant so as to have $g(y) \rightarrow \ln y$ as $\alpha \uparrow 1$, therefore,

$$
g(y)=\frac{y^{\alpha-1}-1}{(\alpha-1) \Gamma(2-\alpha)}+\text { const }
$$

\section{References}

1. Anh, V.V.. and Leonenko, N.. N.; Scaling laws for fractional diffusion-wave equations with singular initial data, Statistics and Probability Letters, 2000 (48), pp 239-252

2. Bakai, E; Fractional Fokker-Planck equatin, solutions and applications, Physical review E, 2001 (63), pp 1-17

3. Caputo, M.; Linear model of dissipation whose Q is almost frequency dependent II, Geophys. J. R. Ast. Soc, 1967 (13), pp 529-539

4. Carrol, R.; On quantum potential, Application Analysis, 2005 (84, No. 11), 1117-1149

5. Decreusefond, L. and Ustunel, A.S.; Stochastic analysis of the fractional Brownian motion, Potential Anal., 1999 (10), pp 177-214

6. Djrbashian, M. M. and Nersesian, A.B.; Fractional derivative and the Cauchy problem for differential equations of fractional order (in Russian), Izv. Acad. Nauk Armjanskoi SSR, 1968 (3), No 1, pp 3-29

7. Doubleday, G.; On linear birth-death processes with multiple births, Mathematical biosciences, 1973 (17), pp 43-56

8. Duncan, T.E., Hu, Y., and Pasik-Duncan, B.; Stochastic calculus for fractional Brownian motion, I. Theory, SIAM J. Control Optim. 2000 (38), pp 582-612

9. El Naschie, MS; A review of E infinity theory and the mass spectrum of high energy particle physics, Chaos, Solitons and Fractals, 2004 (19), pp 209-236

10. El-Sayed, A.; Fractional order diffusion-wave equation, Int. J. Theor. Phys., (1996 (35), pp 311322

11. Frieden, B. R.; Physics from Fisher Information, Cambridge University Press, Cambridge, 2000

12. Grössing, G.; Quantum Cybernetics, Springer, Berlin, 1957

13. Hanyga, A.; Multidimensional solutions of time-fractional diffusion-wave equations, Proc. R Soc. London, A, 2002 (458), pp 933-957

14. Hu, Y. and Øksendal, B.; Fractional white noise calculus and applications to finance, Infinite Dim. Anal. Quantum Probab. Related Topics 62003 (6), 1-32

15. Itô, K.; On stochastic differential equations, Mem. Amer. Soc., 1951 (4)

16. Jumarie, G.; A Fokker-Planck equation of fractional order with respect to time, Journal of Math. Physics, 33, 1992 (10), pp 3536-3542

17. Jumarie, G.; Stochastic differential equations with fractional Brownian motion input, Int. J. Syst. Sc., 1993(6), pp 1113-1132

18. Jumarie, G.; Fractional Brownian motion with complex variance via random walk in the complex plane. Applications, Chaos, Solitons and Fractals, 2000 (11), No 7, pp 1097-1111

19. Jumarie, G.; Maximum Entropy, Information without Probability and Complex Fractals, 2000, Kluwer (Springer), Dordrecht 
20. Jumarie, G.; Schrödinger equation for quantum-fractal space-time of order $\mathrm{n}$ via the complexvalued fractional Brownian motion, Intern. J. of Modern Physics A, 2001 (16), No. 31, pp 50615084

21. Jumarie, G.; Further results on the modelling of complex fractals in finance, scaling observation and optimal portfolio selection, Systems Analysis, Modelling Simulation, 45, 2002 (10), pp 14831499

22. Jumarie, G.; Fractional Brownian motions via random walk in the complex plane and via fractional derivative. Comparison and further results on their Fokker-Planck equations, Chaos, Solitons and Fractals, 2004 (4), pp 907-925

23. Jumarie, G.; On the representation of fractional Brownian motion as an integral with respect to $(d t)^{\alpha}$, Applied Mathematics Letters, 2005 (18), pp739-748

24. Jumarie, G.; On the solution of the stochastic differential equation of exponential growth driven by fractional Brownian motion, Applied Mathematics Letters, 2005 (18), pp817-826

25. Jumarie, G.; A non-random variational approach to stochastic linear quadratic Gaussian optimization involving fractional noises (FLQG), J. Appl. Math. And Computing, 2005 (1-2), pp 19-32

26. Kober, H.; On fractional integrals and derivatives, Quart. J. Math. Oxford, 1940 (11), pp 193-215

27. Kolwankar, KM.; Gangal AD.; Holder exponents of irregular signals and local fractional derivatives, Pramana J. Phys, 1997 (48),pp 49-68

28. Kolwankar KM., Gangal AD.; Local fractional Fokker-Planck equation,, Phys. Rev. Lett., 1998 (80), pp 214-217

29. Letnivov, A.V.; Theory of differentiation of fractional order, Math. Sb., 1868 (3), pp 1-7

30. Liouville, J.; Sur le calcul des differentielles à indices quelconques(in french), J. Ecole Polytechnique, 1832 (13), p 71

31. Mandelbrot, B.B. and van Ness, J.W.; Fractional Brownian motions, fractional noises and applications, SIAM Rev., 1968 (10), pp 422-437

32. Mandelbrot, B.B. and Cioczek-Georges, R.; A class of micropulses and antipersistent fractional Brownian motions, Stochastic Processes and their Applications, 1995 (60), pp 1-18

33. Mandelbrot, B.B. and Cioczek-Georges, R.; Alternative micropulses and fractional Brownian motion, Stochastic Processes and their Applications, 1996 (64), pp 143-152

34. Nelson, E.; Quantum Fluctuations, 1985, Princeton University Press, Princeton, New Jersey

35. Nottale, L.; Fractal space-time and microphysics, 1993, World Scientific, Singapore

36. Nottale, L.; Scale-relativity and quantization of the universe I. Theoretical framework. Astronm Astrophys, 1997 (327), pp 867-889

37. Nottale, L.; The scale-relativity programme, Chaos, Solitons and Fractals, 1999, (10), No 2-3, pp 459-468

38. Olavo, L.S.F.; Foundations of quantum mechanics: The connection between QM and the central limit theorem, Foundations of Physics, 2004 (34), No. 6, pp 891-935

39. Ord, G.N., and Mann R.B.; Entwined paths, difference equations and Dirac equations, Phys. Rev A, 2003 (67): 0121XX3

40. Osler, T. J., Taylor's series generalized for fractional derivatives and applications, SIAM. J. Mathematical Analysis, 1971 ( 2), No 1, pp 37-47

41. Ramakrishman, A. and Srinivasan, S.K.; On age distribution in population growth, Bull. Math. Biophys, 1950 (20), pp 289-308

42. Shawagfeh, N.T.; Analytical approximate solutions for nonlinear fractional differential equations, Appl. Math. And Comp., 2002 (131), pp 517-529

43. Stratonovich, R.L.; A new form of representing stochastic integrals and equations, J. SIAM. Control, 1966 (4), pp 362-371

44. Wyss, W.; The fractional Black-Scholes equation, Fract. Calc. Appl. Anal., (2000 (3), No 1, pp 51-61 